\title{
$R$-band imaging of the host galaxies of RGB BL Lacertae objects
}

\author{
K. Nilsson ${ }^{1}$, T. Pursimo ${ }^{1}$, J. Heidt ${ }^{2}$, L. O. Takalo ${ }^{1}$, A. Sillanpää ${ }^{1}$, and W. Brinkmann ${ }^{3}$ \\ 1 Tuorla Observatory, Väisäläntie 20, 21500 Piikkiö, Finland \\ 2 Landessternwarte Heidelberg, Königstuhl, 69117 Heidelberg, Germany \\ ${ }^{3}$ Max-Planck-Institut für Extraterrestrische Physik, Giessenbachstrasse, 85740 Garching, Germany
}

Received 23 August 2002 / Accepted 16 December 2002

\begin{abstract}
We present optical $R$-band images of 100 BL Lacertae objects in the ROSAT-Green Bank (RGB) sample obtained at the Nordic Optical Telescope (NOT). For 62 objects we could not find any previously published images in the literature, of these 35 are new BL Lacs discovered during the RGB BL Lac survey. We were able to resolve the host galaxy in $62 \%$ of the objects. We analyze the host galaxies using a two-dimensional fitting procedure and tabulate the best-fit core magnitude and host galaxy parameters (magnitude, effective radius, ellipticity, position angle and shape parameter $\beta$ ) for each object. With two exceptions, all objects are better fit by a model representing an elliptical galaxy $(\beta=0.25)$ than by a disk galaxy model $(\beta=1.0)$. In the two exceptions the disk host fit is only marginally better than the elliptical host fit, however, and we do not find a single clear example of a disk-dominated host galaxy. The host galaxies have an average brightness $M_{R}=-23.9 \pm 0.8$ and average effective radius $r_{\text {eff }}=13.2 \pm 0.8 \mathrm{kpc}$. Their bulk properties are indistinguishable from normal elliptical and radio galaxies. The median $\beta$ is 0.18 , well below the value 0.25 that is normally used to describe ellipticals. However, the difference may be caused by a selection effect due to the dependence of $\beta$ on host galaxy luminosity.
\end{abstract}

Key words. BL Lacertae objects: general - galaxies: nuclei - galaxies: structure

\section{Introduction}

BL Lacertae objects (BL Lacs) are a subclass of active galactic nuclei characterized by almost featureless optical continuum, high optical polarization $(p>3 \%)$ and variability over the whole electromagnetic spectrum. These properties are believed to be caused by a relativistic jet aligned close to the line of sight. Doppler boosting and relativistic beaming enhance the nuclear radiation considerably and make the detection of the host galaxy and the line emission very difficult.

Previous studies (e.g. Abraham et al. 1991; Stickel et al. 1993; Wurtz et al. 1996; Falomo \& Kotilainen 1999; Scarpa et al. 2000a; Urry et al. 2000; Pursimo et al. 2002) have shown that BL Lacs lie in the nuclei of luminous $\left(M_{R} \sim-23.7\right)$ and large $\left(r_{\text {eff }} \sim 10 \mathrm{kpc}\right)$ elliptical galaxies. Their bulk properties appear to be indistinguishable from those of the host galaxies of Fanaroff-Riley (FR) type I radio sources (Fanaroff \& Riley 1974), supporting the unifying view that BL Lacs are FR I radio sources seen almost along the direction of the jet (Urry \& Padovani 1995). Further support for the unifying view comes from the fact that the extended radio luminosities and emission line luminosities seem to be similar in both types of objects (see Urry \& Padovani 1995).

So far any possible connection between the nuclear properties and the host galaxy properties has remained elusive. Wurtz et al. (1996) found no difference between the host galaxies of

Send offprint requests to: K. Nilsson, e-mail: kani@astro.utu.fi
$\mathrm{X}$-ray and radio selected BL Lacs. A similar conclusion was reached by Urry et al. (2000), who found no difference between the host galaxies of "red" and "blue" (i.e. low frequency peaked and high frequency peaked) BL Lacs in their HST study of 110 objects. On the other hand, the broad emission line AGN studied by Schade et al. (2000) showed a weak but significant correlation between the nuclear brightness and the host galaxy brightness. A similar weak correlation was found for X-ray selected BL Lacs by Falomo \& Kotilainen (1999) and for the radio selected 1 Jy BL Lacs by Pursimo et al. (2002), although in both papers it was noted that this correlation may be a result of selection effects, e.g. missing faint nuclei in very luminous hosts.

Since the central black hole is ultimately responsible for the production of nuclear activity, the possibility of a nucleus-host galaxy connection has become more appealing recently with the findings that many global properties of galaxies seem to correlate with the central black hole mass. Firstly, Magorrian et al. (1998) found that the kinematic data of 36 nearby galaxies indicate the presence of a massive dark object in the center of the galaxies and that the mass of the dark object correlates with the bulge luminosity and mass. Recent studies of this relationship obtain $M_{B H} \sim 0.001 M_{\text {bulge }}$ (McLure \& Dunlop 2002; Merritt \& Ferrarese 2001). Secondly, the central velocity dispersion of the bulge $\sigma_{0}$ has been found to exhibit a tight correlation with the central black hole mass (Ferrarese \& Merritt 2000, Gebhardt et al. 2000). Falomo et al. (2002) have recently applied this correlation to 7 BL Lacs and found the black 
hole masses to be in the range of $\log \left(M_{B H}\right)=7.7-8.9 M_{\odot}$. Furthermore, Wu et al. (2002) estimated $\sigma_{0}$ for 92 AGN with elliptical hosts using the fundamental plane and found a range of masses of $\log \left(M_{B H}\right)=7.5-9.0 M_{\odot}$. Finally, Graham et al. (2001) discovered a correlation between the bulge light profile and the central black hole mass that appears to be even tighter than the correlation with central velocity dispersion. Fitting Sérsic (1968) $1 / n$ light profiles to the bulge light distributions they found $\log \left(M_{B H}\right)=6.42+2.93 \log (n)$ with a 0.32 dex scatter in $\log \left(M_{B H}\right)$.

Given the above relations it seems that an accurate analysis of the host galaxy light profile might provide a way to estimate the central black hole masses in BL Lacs and to study the dependence of nuclear parameters on the host galaxy parameters. This kind of analysis can also be used to test the unified scheme, the relation between BL Lacs and nonactive galaxies and the cosmological evolution of the BL Lac population. With these goals in mind we started an observing program of several BL Lac samples at the Nordic Optical Telescope (NOT). Part of these observations have already been published (Heidt et al. 1999a; Nilsson et al. 1999a; Pursimo et al. 2002). Here we present the images and fitting results for the RGB sample. A more detailed statistical analysis will follow in a subsequent paper.

Throughout the paper we assume $H_{0}=50 \mathrm{~km} \mathrm{~s}^{-1} \mathrm{Mpc}^{-1}$ and $q_{0}=0.0$ when computing absolute parameters.

\section{The sample}

The ROSAT-Green Bank (RGB) sample of BL Lacertae objects was formed by Laurent-Muehleisen et al. (1999, hereafter LM99). The ROSAT All-Sky Survey (Voges 1992) was first correlated with the $5 \mathrm{GHz}$ Green Bank radio survey (Gregory et al. 1996) by selecting sources with $\Delta_{r x}<$ 100 arcsec (Brinkmann et al. 1996). This initial catalog of 2127 sources was then observed at the VLA for accurate radio positions, which produced a list of 1567 reliable RASS-VLA matches. Optical identification was made using the Automatic Plate Measuring (APM) scans of the Palomar Observatory Sky Survey (POSS) I plates (971 reliable RASSVLA-POSS I matches were found) after which optical spectroscopy was used to classify the brightest $(\mathrm{O} \leq 18.5)$ objects (Laurent-Muehleisen et al. 1998, hereafter LM98). Of the 594 classified sources 53 turned out to be BL Lacs, of which 38 were new discoveries. Combining these BL Lacs with previously known BL Lacs in the RGB catalog yielded the final RGB BL Lac catalog of 127 objects.

The catalog is triply limited (radio, optical, $\mathrm{X}$-ray) but not complete, although a complete subsample of 33 objects was drawn from the sample (LM99). The flux limits in the three different bands depend on various factors: the radio limit is $\sim 15 \mathrm{mJy}$ for declinations above $30^{\circ}$ and $\sim 24 \mathrm{mJy}$ below. The $\mathrm{X}$-ray limit is set by the RASS to a few times $10^{-13} \mathrm{erg} \mathrm{cm}^{-2} \mathrm{~s}^{-1}$ in the $0.1-2.4 \mathrm{keV}$ band depending on spectral shape and amount of galactic absorption. The optical limit is less well defined. The complete sample has the limit $O<18.0 \mathrm{mag}$, but the rest of the sample has no clearly defined optical limit. Given the above limits, the completeness of the sample is hard to assess (LM99).

In the $\alpha_{o x}-\alpha_{r o}$ color-color diagram the RGB BL Lacs cover a larger area than previous samples made using a single selection frequency. Given this wide scatter in broadband nuclear properties the RGB catalog provides an excellent sample to study the nucleus - host galaxy connection. Another interesting feature of the RGB sample is the inclusion of borderline objects, termed as BL Lac "candidates" in LM99. These objects do not adhere to the rather strict definition used by Stocke et al. (1991), namely that all emission lines must have $W_{\lambda}<5 \AA$ and the spectral drop at the $\mathrm{Ca} \mathrm{H} \& \mathrm{~K}$ lines near $4000 \AA\left(\mathrm{Br}_{4000 \AA}=\right.$ $(f(4000+)-f(4000-)) / f(4000+))$ is smaller than $25 \%$. In addition to objects satisfying these criteria LM99 included also objects with $25 \%<\mathrm{Br}_{4000 \AA}<40 \%$ as candidate BL Lacs. The candidate objects were also allowed to have $W_{\lambda}>5 \AA$ if it was smaller than $a \mathrm{Br}_{4000 \AA}$-dependent limit prescribed by Marchã et al. (1996). The latter criterion is still somewhat arbitrary, but it is less dependent of the dilution of nuclear light by the host galaxy and thus includes objects with optically fainter nuclei with BL Lac type characteristics. Additionally, any object denoted as candidate by previous authors or objects whose classification was based on a low resolution ( $80 \AA$ ) spectrum was termed as candidate. There are altogether 27 candidates in the RGB BL Lac sample, 21 of which were observed during this study.

One unfortunate deficit of the RGB BL Lac sample is that the redshifts are known for only $\sim 50 \%$ of the sample and some redshifts are highly uncertain. Since the determination of the redshift requires detection of nuclear emission lines or absorption lines of the host galaxy, it is possible that objects with strong emission lines (for a BL Lac object) and/or bright host galaxies are preferred among the objects with known $z$. Given the possibility of these biases one should thus be careful when interpreting the results.

\section{Observations}

The observations were carried out at the Nordic Optical Telescope (NOT) ${ }^{1}$ on La Palma, Spain during several observing runs in 1995-2002. We used three different instruments, whose characteristics are listed in Table 1. HiRAC and StanCam are direct imaging cameras with field of view of $3.8 \times 3.8$ and $3^{\prime} \cdot 0 \times 3{ }^{\prime} 0$, respectively, whereas ALFOSC is a focal reducer giving a field of view of 6.5 $\times 6.5$.

We obtained (Cousins) $R$-band images for 100 objects, and additional $B, V$ or $I$-band images for $\sim 40$ objects. Here we discuss the $R$-band images only. The individual exposures of the BL Lacs were kept short enough as not to saturate the nucleus. For bright objects $(R<15)$ we obtained several exposures of $15-150 \mathrm{~s}$, which were then combined. For fainter objects a few exposures of 300-900 s were sufficient. The total exposure times vary from 90 to $6000 \mathrm{~s}$ depending on the relative prominence of the host galaxy. Table 3 gives the log of observations.

\footnotetext{
1 Nordic Optical Telescope is operated on the island of La Palma jointly by Denmark, Finland, Iceland, Norway, and Sweden, in the Spanish Observatorio del Roque de los Muchachos of the Instituto de Astrofisica de Canarias.
} 
Table 1. The instruments used in this study.

\begin{tabular}{lclll}
\hline \hline Instrument & pixels & $\begin{array}{l}\text { pix. scale } \\
{\left[\text { pix. }^{-1}\right]}\end{array}$ & $\begin{array}{l}\text { gain } \\
{\left[\mathrm{e}^{-} / \mathrm{ADU}\right]}\end{array}$ & $\begin{array}{l}\text { RON } \\
{\left[\mathrm{e}^{-}\right]}\end{array}$ \\
\hline HiRAC & $2048^{2}$ & 0.11 & 1.3 & 6.2 \\
StanCam & $1024^{2}$ & 0.176 & 1.7 & 6.5 \\
ALFOSC & $2052^{2}$ & 0.189 & 1.0 & 6.5 \\
\hline
\end{tabular}

Columns 1 and 2 give the RGB name and the name in other catalogs, Col. 3 indicates whether the object is a BL Lac candidate in LM98, Col. 4 whether the object was a new BL Lac in LM98, Col. 5 gives the galactic extinction in the $R$-band from Schlegel et al. (1998), obtained through NED ${ }^{2}$, Col. 6 the observing date, Col. 7 the instrument, Col. 8 the total integration time, Col. 9 the seeing and Col. 10 gives references to previous observations that have been published since 1990 .

We have already published data for $14 \mathrm{BL}$ Lac objects in Table 3. Pursimo et al. (2002) analyzed the host galaxies of $1 \mathrm{Jy}$ BL Lacs. Of the 9 objects common to this study 8 are re-analyzed here with our 2-dimensional decomposition procedure. We excluded $0721+713$ from this study due to the fact that no suitable PSF stars were available in the frame. Images of $1745+398$ and $1806+468$ have been published before by Nilsson et al. (1997, 1999b), but no host galaxy analysis was made. The images and fitting results for $1104+382$, $1442+120,1653+397,1743+195$ and $2039+523$ are reproduced here from our previous studies for completeness (Heidt et al. 1999a, 1999b; Nilsson et al. 1999a). We have indicated in Table 3 the objects for which we have published data before with the reference to the original publication.

The images were reduced in a standard way by subtracting the bias, dividing by twilight flat-fields and removing the cosmic ray hits using IRAF procedures. For ALFOSC we used flat-fields obtained at different position angles of the instrument to remove the background nonuniformity caused by scattered light. Generally a better than $1 \%$ uniformity of the sky background over the entire field was achieved. The photometric calibration was made by observing several photometric standard stars in Landolt (1992) during the night. When the weather conditions were not photometric we used calibrated comparison stars in the same field as the object (Fiorucci \& Tosti 1996; Fiorucci et al. 1998; Villata et al. 1998).

\section{Analysis}

\subsection{PSF variability}

It is well known that an accurate determination of the PSF is crucial to the nucleus/host galaxy separation, especially for marginally resolved objects. The best results are obtained by using observed stars in the same field as the BL Lac under study. In this way one can circumvent problems associated with changing seeing conditions and/or tracking errors. However,

2 The NASA/IPAC Extragalactic Database (NED) is operated by the Jet Propulsion Laboratory, California Institute of Technology, under contract with the National Aeronautics and Space Administration. aberrations in the imaging system can introduce PSF changes over the field of view and thus degrade the results.

We made an examination of the PSF shape ( $F W H M$, ellipticity and PA) over the field of all three instruments. This examination revealed that in HiRAC and StanCam the PSF is very stable over the field of view. In ALFOSC, however, the PSF shape is changing considerably: in typical frames the FWHM changes $\sim 7 \%$, the ellipticity by $\sim 0.04$ and the position angle more than 90 degrees over the field of view. These changes are caused by the re-imaging optics of ALFOSC that introduce a curved focal plane and astigmatism (Rasmussen 1996; Andersen, priv. comm.).

We investigated whether the PSF changes could be modeled such that they could be used in our simulations (Sect. 4.3). We decided to concentrate on the lowest order aberrations that cause the change of $F W H M, \epsilon$ and PA over the field. The star images contain also higher order aberrations (e.g. coma) but to model these would complicate the task considerably with little gain since the low-order aberrations usually dominate. ALFOSC produces also ghost images that may contribute to the PSF variability, but their brightness is very low $(<0.05 \%$ of peak intensity).

We experimented with different ways to model the PSF and ended up with a model that consists of two parts, an atmospheric part and an aberration part. The atmospheric part is modeled with an azimuthally symmetric Moffat profile described by three parameters, the magnitude, Moffat $\beta$ and $F W H M$. This part represents the atmospheric smearing and it is assumed to be constant over the field. The aberration part is described via the 6 lowest order Zernike polynomials $Z_{k}(r, \theta)$, $k=1 . .6$, of which the first 3 terms $Z_{1.3}$ (the "piston", tip and tilt terms, respectively) have no effect on the PSF shape. The $Z_{4}$-term is associated with defocus and $Z_{5}$ and $Z_{6}$ with astigmatism. We first compute the phase error $\phi$ at the pupil from

$$
\phi(r, \theta)=\sum_{i=2}^{6} a_{i} Z_{i}(r, \theta)
$$

parametrized by the Zernike coefficients $a_{2 \ldots 6}\left(a_{1}=0\right)$, multiply it with a mask containing the secondary mirror and secondary support and finally Fourier transform to produce the aberrated image corresponding to $a_{2 \ldots 6}$. The computations are made assuming that the light is monochromatic with $\lambda=$ $640 \mathrm{~nm}$. The aberrated image is then convolved with the atmospheric part and rebinned to the instrument resolution to yield the final model image. Using an iterative Levenberg-Marquardt loop we fit this model to each star in the field by $\chi^{2}$ minimization. To improve the convergence to the global minimum we use a pyramidal recursive algorithm similar to Iglesias (1998). We make two passes through the stars in each field. During the first pass the Moffat $\beta$ and $F W H M$ of the atmospheric profile are free parameters and during the second pass, they are held constant at the median values from the first pass. The total number of free parameters is 8 during the first pass and 6 (magnitude, $a_{2}-a_{6}$ ) during the second pass. From 5 to 60 (typically 10-15) stars per field were fit with the above fitting procedure depending on the number of suitable stars. 

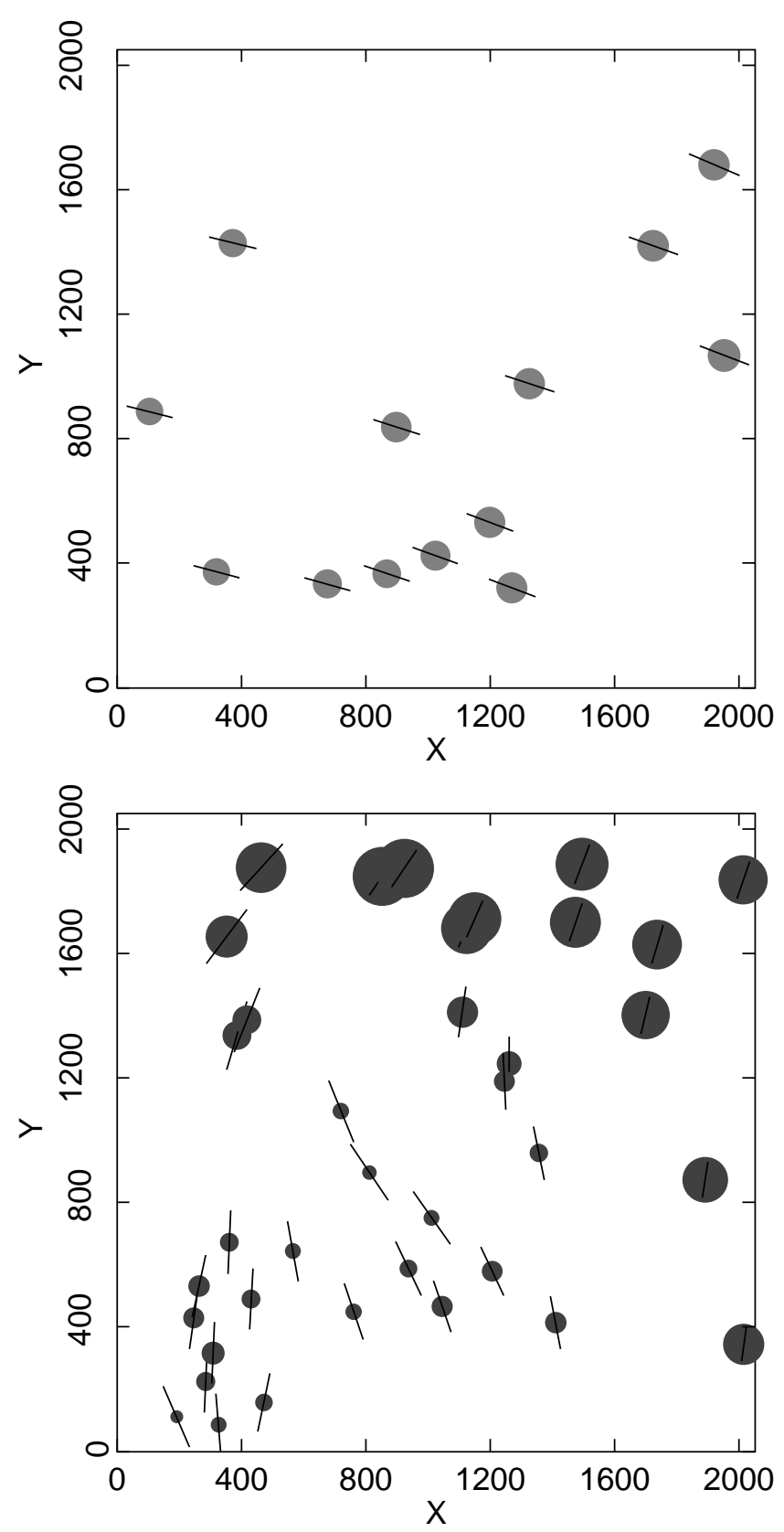

Fig. 1. Two examples of the PSF variability over the field of view in HiRAC (upper panel) and ALFOSC (lower panel). Each symbol corresponds to a star in the field. The diameter of the symbol is proportional to the fitted $a_{4}$ (defocus) coefficient. The direction and length of the lines are proportional to the $a_{5}$ and $a_{6}$ (astigmatism) coefficients. Note the difference in PSF variations across the field of view of the two instruments.

In Fig. 1 we show two examples of the results obtained with this fitting procedure. Each circle/line pair corresponds to a star in the two particular BL Lac fields shown here as an example. Since the NOT is an alt-az telescope, the alignment of the telescope optics changes with respect to the instrument from object to object and each field gives slightly different results. The two cases in Fig. 1 are typical, however, and illustrate the difference of PSF variability in HiRAC and ALFOSC.
Our analysis of the PSF changes across the field of view confirms our initial finding that the PSF variations are more severe in ALFOSC than in HiRAC and StanCam. The focal planes of HiRAC and StanCam are flat but slightly tilted with the direction of the tilt changing from object to object. In ALFOSC the focal plane is curved with possible tapering in the edges, and relatively constant from object to object. It is qualitatively similar to the focal plane map given in Rasmussen (1996). The average peak-to-peak variation in $a_{4}$ is $\sim 1.7$, which corresponds to a $0.54 \lambda$ phase error or

$$
0.54 \cdot 8 \lambda F=110 \mu \mathrm{m}
$$

in the focal plane, where $F$ is the focal ratio (6.4 for ALFOSC). This is slightly smaller than the value $150 \mu \mathrm{m}$ quoted in Rasmussen (1996), but reasonable given the number of assumptions in the model and possible color effects and a tilt of the focal plane.

This procedure works reasonably well when the aberrations are roughly constant, i.e. when the observations have been made during a short interval and/or when the instrument has not rotated strongly. Occasionally, bad results are obtained even though the optical alignment should have been stable during the exposures. These may be cases where tracking errors or higher order aberrations contribute significantly to the PSF shape. It was also found that a good $S / N$ is required for optimal results and thus the noise in $a_{4}-a_{6}$ is higher in fields populated mostly by faint stars.

\subsection{Method of analysis}

The host galaxy fitting procedure is described in detail in Nilsson et al. (1999a). A 2-dimensional model is fitted to the observed image via $\chi^{2}$ minimization using a LevenbergMarquardt loop. The model consists of two components, the BL Lac core and the host galaxy. The core is parametrized by its position $\left(x_{\mathrm{c}}, y_{\mathrm{c}}\right)$ and magnitude $m_{\mathrm{c}}$, whereas the host galaxy is parametrized by position $\left(x_{\mathrm{g}}, y_{\mathrm{g}}\right)$, total magnitude $m_{\mathrm{g}}$, effective radius $r_{\text {eff }}$, ellipticity $\epsilon$, position angle PA and shape parameter $\beta$ (equal to $1 / n$ in the Sérsic (1968) model). The surface brightness of the galaxy is obtained from

$I(r)=I\left(r_{\mathrm{e}}\right) \operatorname{dex}\left\{-b_{\beta}\left[\left(\frac{r}{r_{\mathrm{e}}}\right)^{\beta}-1\right]\right\}$,

where $b_{\beta}$ is a $\beta$-dependent constant defined such that $r_{\mathrm{e}}$ always encircles half of the total light.

The model is convolved with the PSF determined by averaging 2-3 stars in the field, that were comparable in brightness to the BL Lac. In a few cases bright enough stars were not available, which resulted in a lower $S / N$ PSF. This may have caused a failure to detect a weak host galaxy or resulted in less accurate host galaxy parameters in some cases. However, our error simulations (Sect. 5) take into account the effect of the noisy PSF, so cases where this effect might be significant can be recognized. We also tried to improve the PSF using our modeling of the PSF changes across the field in the previous section. In principle, if sufficiently many stars in the field could be modeled, one could predict the shape of the PSF at the object's location. Tests on 
several fields showed that the PSF can be improved when the exposures have been made over a short time period, i.e. when the optical aberrations are almost constant, and when sufficient number of stars (15-20) are available in the field. Since these conditions are not always met, we decided not to correct the PSF in order to treat all objects in the same way.

Before fitting, the background level was estimated by averaging measurements made in rectangular areas around the BL Lac and subtracted. In cases where a background gradient was present a low-order surface was fit to the background pixels and subtracted. All overlapping objects were masked out prior to fitting. In some cases we iterated between fitting the BL Lac and a nearby object and subtracting either of the objects before fitting the other. Sometimes it was not possible to completely mask a nearby source because it extended over the BL Lac core. We note in Sect. 5.2 the cases where we suspect that nearby sources have affected the results. The fit was limited to a circular region with such a radius that the minimum surface brightness of the object was $\sim 3-5$ times higher than the uncertainty of the background level, (typically $\mu_{\mathrm{obj}} \sim 25 \mathrm{mag} / \mathrm{sq}$ arcsec at the outer radius).

The amount of information obtainable of the host galaxies depends on the distance of the object and on the nuclear dominance $F_{\text {nucl }} / F_{\text {host }}$, both of which vary considerably over the sample. The minimum we wanted to achieve is to get an estimate of the magnitude and size of the host galaxy. Therefore we made two types of fits depending on the relative brightness of the host galaxy. When the host galaxy was reasonably strong compared to the nucleus, we fitted three galaxy models with $\beta=0.25$ (de Vaucouleurs profile), $\beta=1.0$ (exponential profile) and $\beta$ as a free parameter. The total number of free parameters was 9 or 10. If the host galaxy was very weak (more than $\sim 2$ mag fainter than the nucleus), we fitted only two models $(\beta=0.25$ and 1.0$)$ keeping the core and host galaxy position fixed and $\epsilon=\mathrm{PA}=0$. The number of free parameters was three $\left(m_{\mathrm{c}}, m_{\mathrm{g}}\right.$ and $\left.r_{\mathrm{e}}\right)$ in these cases. There were 7 such cases in the sample. The effective radii obtained with these two methods are not directly comparable, but they can be made such by multiplying the effective radii of the first type fits by $\sqrt{1-\epsilon}$.

\subsection{Simulations}

We have made simulations to test the modeling algorithm and its sensitivity to different noise sources, including photon and readout noise, PSF variability and background determination error. We made 14 simulation runs corresponding to different contributions of the above noise sources. In each run we created six sets of 30 simulated images of an adopted "standard" BL $\mathrm{Lac}+$ host galaxy (elliptical, $M_{R}=-23.7$ and $r_{\mathrm{eff}}=10 \mathrm{kpc}$ ). The six sets corresponded to two cases of nuclear brightness, $m_{\text {core }}=m_{\text {host }}$ and $m_{\text {core }}=m_{\text {host }}-2.5$ and three redshifts $z=0.15,0.3$ and 0.5 . We added the effect of galactic extinction ( $A_{R}=0.1$ was assumed), K-correction and passive evolution to the apparent magnitudes of the host galaxy. All simulated images corresponded to a $900 \mathrm{~s}$ exposure with the HiRAC at 0.7 seeing and a sky background of $21.5 \mathrm{mag} / \mathrm{sq}$ arcsec.
Each simulated image was constructed as follows: first a noise-free core + host galaxy image was made. Then $a_{4}-a_{6}$ in Eq. (1) were drawn from an uniform distribution and the corresponding PSF was created, after which the model was convolved with this PSF. Then photon and readout noise plus a small offset from a zero-mean Gaussian distribution that represents the sky determination error were added to the simulated image. Prior to the fitting the defocus coefficient $a_{4}$ was changed by a small amount and a second PSF was created. Photon and readout noise were added to this second PSF, after which it was used as the PSF model in the fit. Each simulated image was thus convolved with a different PSF and fitted with a another PSF that differed from the first PSF with respect to the defocus coefficient $a_{4}$. This simple approach produced residuals that closely resemble the "quadrupole-like" residuals seen in the data (see e.g. $0929+502$ in Fig. 10). For the simulated fits we used the same procedure as for the real data (Sect. 4.2). After each fit the mean values and standard deviations we logged.

In the noiseless case the program found the correct parameters to a high accuracy. As expected, the more noise was added the higher the scatter in the fitted parameters became. The most significant finding was that noise in the PSF biases the results if the host galaxy is apparently small (i.e. at $z>0.3$ ). One obtains too small and too faint host galaxies from the fits when using a low $S / N$ PSF star. To avoid this bias one should use a PSF star that is 1 to 2 magnitudes brighter than the object or, alternatively, average several stars in the field. Noise in the BL Lac image also biases the results in a similar way, but in this case the bias is $<10 \%$ for $r_{\mathrm{eff}}$ and $<0.05 \mathrm{mag}$ for $m_{\mathrm{g}}$ at $z \geq 0.3$ for an exposure of $900 \mathrm{~s}$. Well-resolved sources $(z=0.15)$ are virtually free from the above biases. PSF or background determination errors increase the scatter in parameter distributions, but do not bias the average results.

\section{Results}

The results of the host galaxy fits are given in Tables 4 and 5 . Table 4 gives the fit results for the de Vaucouleurs model and for the unresolved objects. Table 5 gives the results for the free $\beta$ model. As explained above, the free $\beta$ model was fitted only to objects that were subjectively judged to be resolved well enough (55 objects).

The errors of the fitted parameters were estimated via simulations. For each object we created 30-50 simulated images that corresponded to the best-fitting parameters and performed the fits in a way similar to the actual fits. To the simulated images we added photon and readout noise, background uncertainty and PSF noise in a way similar to the test simulations (Sect. 4.3). The amount of defocus needed to introduce the PSF errors was determined by examining the residuals of the actual fits and adjusting the defocus to match the observed residuals. It is clear that this simple approach does not take into account all elements of the PSF variability, but in most cases the residuals are very similar to the residuals seen in the actual data. The residuals are more complex in cases where the exposures have been taken over the course of several hours or during different nights. In these cases the defocus was adjusted to obtain the 
right amplitude of residuals although their exact shape is not correct.

The errors in Tables 4 and 5 give the standard deviation of each parameter in the simulations. To the magnitude errors we have added in square the error of photometric calibration, which is $0.03-0.05$ mag in most cases. Although we have included most noise sources into our simulations, the errors should still be treated as lower limits, since they do not include systematic errors. The latter may arise e.g. when a de Vaucouleurs model does not adequately describe the surface brightness distribution of the host galaxy (and in many host galaxies it clearly does not).

The columns in Table 4 give:

(1) Object name in the RGB list.

(2) Redshift.

(3) Resolved or unresolved? Via simulations we determined that the fitting program converges either towards $r_{\mathrm{eff}} \rightarrow 0$ or $r_{\text {eff }} \rightarrow \infty$ when the host galaxy is below the noise level. Any object producing this kind of result is denoted as "unresolved" in Table 4. For the rest we require that $r_{\text {eff }}>5 \sigma_{\text {reff }}$ for the object to be resolved, where $\sigma_{\text {reff }}$ comes from the error simulations. Given that the error bars are lower limits we decided to use this rather strict limit.

(4) $R$-band magnitude of the BL Lac nucleus. For unresolved objects this comes from a fit with a core component only. The magnitudes are observed magnitudes with no corrections applied.

(5) $R$-band magnitude of the host galaxy. These are, again, observed magnitudes with no corrections applied.

(6) Effective (half-light) radius of the host galaxy in arcsec along the major axis.

(7) Surface brightness at the effective radius of the host galaxy in $\mathrm{mag} / \mathrm{sq}$ arcsec.

(8) Ellipticity of the host galaxy. A dash indicates that the ellipticity was held constant (0.0) during the fit.

(9) Position angle of the host galaxy, measured anticlockwise from the north. A dash indicates that the PA was held constant (0.0) during the fit.

(10) Reduced chi squared of the fit.

(11) The ratio between the $\chi^{2}$ of the exponential model $(\beta=1.0)$ and the $\chi^{2}$ of the de Vaucouleurs model $(\beta=0.25)$.

(12) This column is marked with a Y if a new host galaxy detection has been made in this paper.

The same parameters for the free $\beta$ model are given in Table 5 in addition to the best-fitting $\beta$ in Col. 8 .

In Fig. 2 we show the 1-dim. radial profiles of the 37 objects where we have made a new host galaxy detection. The profiles were extracted from the 2-dim. images using the ellipse task in IRAF. In addition to the observed points (marked with asterisks) we show the seeing-convolved core + host galaxy model (solid line), host galaxy model (dashed line) and the core model (dotted line). In Fig. 10 we show four examples of BL Lac images before and after subtracting the best-fitting 2-dim. model. The field size, lowest contour level (in mag/sq arcsec) and the host galaxy model used in the subtraction are given below each image. Contour interval is $1 \mathrm{mag} / \mathrm{sq}$ arcsec. In Fig. 11 we show similar images of four interesting objects.
Table 6 gives the intrinsic parameters of the host galaxies for objects with known redshift. The meaning of the Cols. is:

(1) RGB name.

(2) The K-correction in the $R$-band from Fukugita et al. (1995).

(3) Core absolute magnitude for the de Vaucouleurs model. For unresolved objects the core magnitude comes from a fit with the core component only. The magnitudes have been corrected for galactic absorption, but no K-correction has been applied (i.e. we have assumed a power law spectrum of the form $\left.I_{v} \propto v^{-1}\right)$.

(4) Host galaxy absolute magnitude for the de Vaucouleurs model. The magnitudes have been corrected for galactic absorption and a K-correction from Fukugita et al. (1995) has been applied.

(5) Host galaxy effective radius in kpc. The values have been multiplied by $\sqrt{1-\epsilon}$, where $\epsilon$ is the host galaxy ellipticity.

(6)-(8) Same as (3)-(5), but for the free $\beta$ model.

There are 5 objects in the sample that have a spectroscopically measured redshift, but no host galaxy was detected. We have estimated an upper limit for their host galaxy luminosity using sets of simulated BL Lac images. Each set consisted of 30 simulations and the brightness of the host galaxy was changed by $0.3 \mathrm{mag}$ between the sets. In all sets we used $r_{\mathrm{eff}}=13 \mathrm{kpc}$ (the average value of the whole sample) and $\beta=0.25$ for the host galaxy. The core magnitude was obtained from the fits with a core component only. We fitted the 30 simulated images with a core + host galaxy model and computed the average fitted effective radius $<r_{\text {eff }}>$ and the rms scatter $\sigma_{\text {reff }}$ for each simulation set. We then plotted $<r_{\text {eff }}>/ \sigma_{\text {reff }}$ against the host galaxy magnitude and the point where this curve fell below $<r_{\text {eff }}>/ \sigma_{\text {reff }}=5.0$ was adopted as the upper limit for the host galaxy magnitude. This upper limit and the corresponding upper limit for the host galaxy luminosity are given in Tables 4 and 6 , respectively.

\subsection{Comparison with previous results}

In Fig. 3 we compare the fitted host galaxy parameters (magnitude and effective radius) to those found in the literature. We found 26 objects common in three studies. All have used a de Vaucouleurs model for the host galaxy. Two of the studies (Falomo \& Kotilainen 1999; hereafter FK99 and Pursimo et al. 2002; hereafter P02) have obtained their data with the NOT, the P02 data are the same as used in this study. Any differences between our results and the $\mathrm{P} 02$ results should thus reflect differences in the analysis method. The third dataset comes from the HST study of Scarpa et al. (2000a; hereafter SU00). All comparison sets have been analyzed with the same 1-dim analysis package, whereas we have used a 2-dim method. To facilitate the comparison we have multiplied our effective radii by $\sqrt{1-\epsilon}$.

For the host galaxy magnitudes we do not find any systematic differences (Fig. 3a). The average difference between our magnitudes and the comparison magnitudes is $0.00 \pm 0.04$ mag with a rms scatter of 0.23 mag. The latter is clearly larger than most of the quoted error bars, which is most likely due to systematic errors not accounted for in the error analyses. 

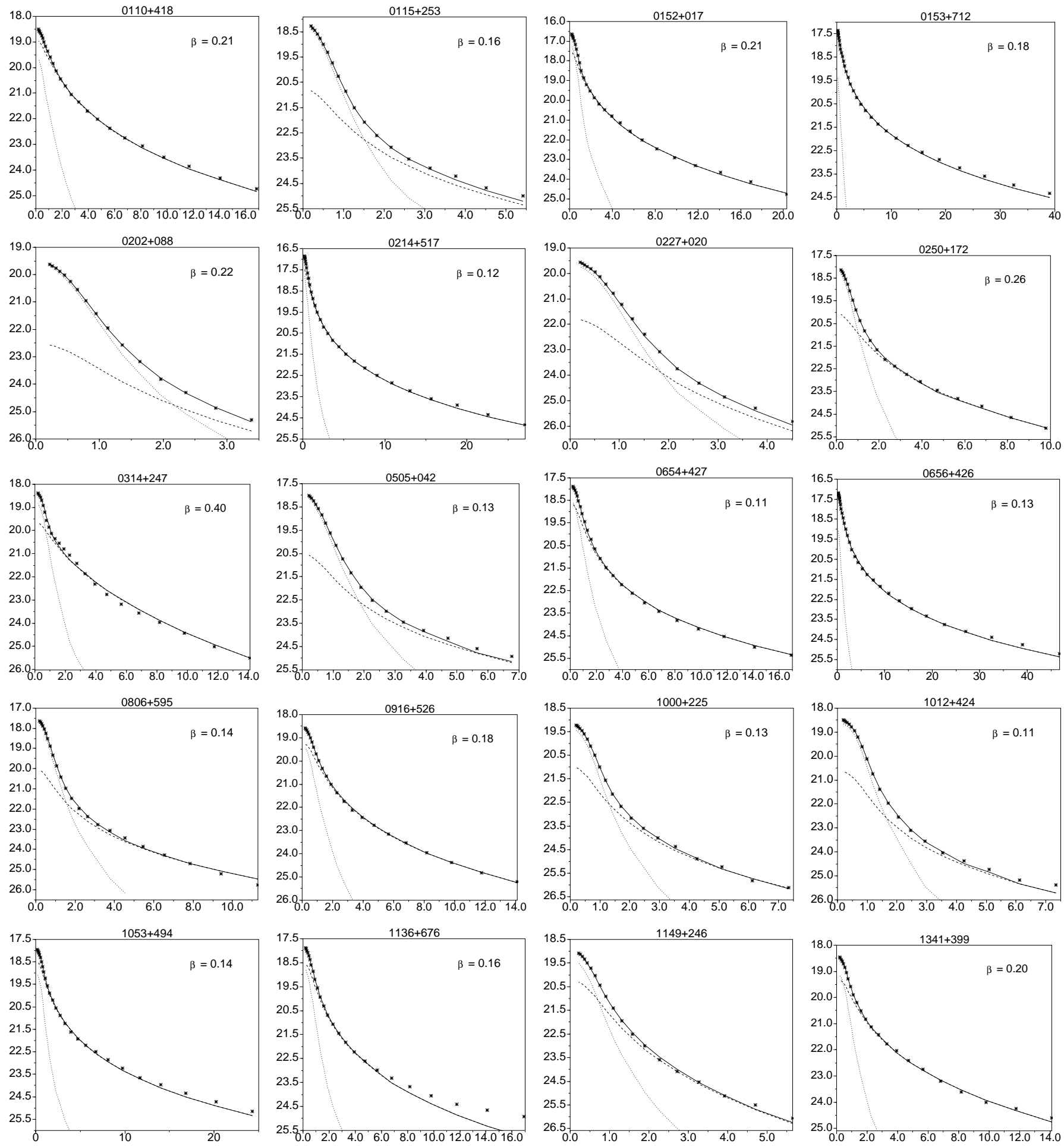

Fig. 2. Radial profiles of the new host galaxy detections. The $x$-axis shows the major axis distance from the nucleus in arcsec and the $y$-axis the $R$-band surface brightness in mag arcsec ${ }^{-2}$. Asterisks mark the observed points, the solid line the core + host galaxy model, dashed line the host galaxy model and the dotted line the core model. The $\beta$ used in the host galaxy model is also given, except when a de Vaucoulerus model $(\beta=0.25)$ has been used.

The average difference to the $\mathrm{P} 02$, who used the same data as we did, is $0.12 \pm 0.04$ mag ( 6 data points) with a 0.09 mag rms scatter. The effective radius seems to be less well determined, especially for large $r_{\text {eff }}$, as can be seen in $3 b$. For objects with $r_{\text {eff }}<5$ arcsec the rms scatter is $1.5 \operatorname{arcsec}$, i.e. $\sim 50 \%$ in most of the sources. For large host galaxies $\left(r_{\mathrm{eff}}>5 \operatorname{arcsec}\right)$ a systematic difference is seen with respect to the HST data, which seem to indicate systematically smaller galaxies than our ground-based images.

The reason for these differences lies probably in the difficulty of accurately determining the scale length in the presence of a bright nucleus. One source of discrepancy could be that 

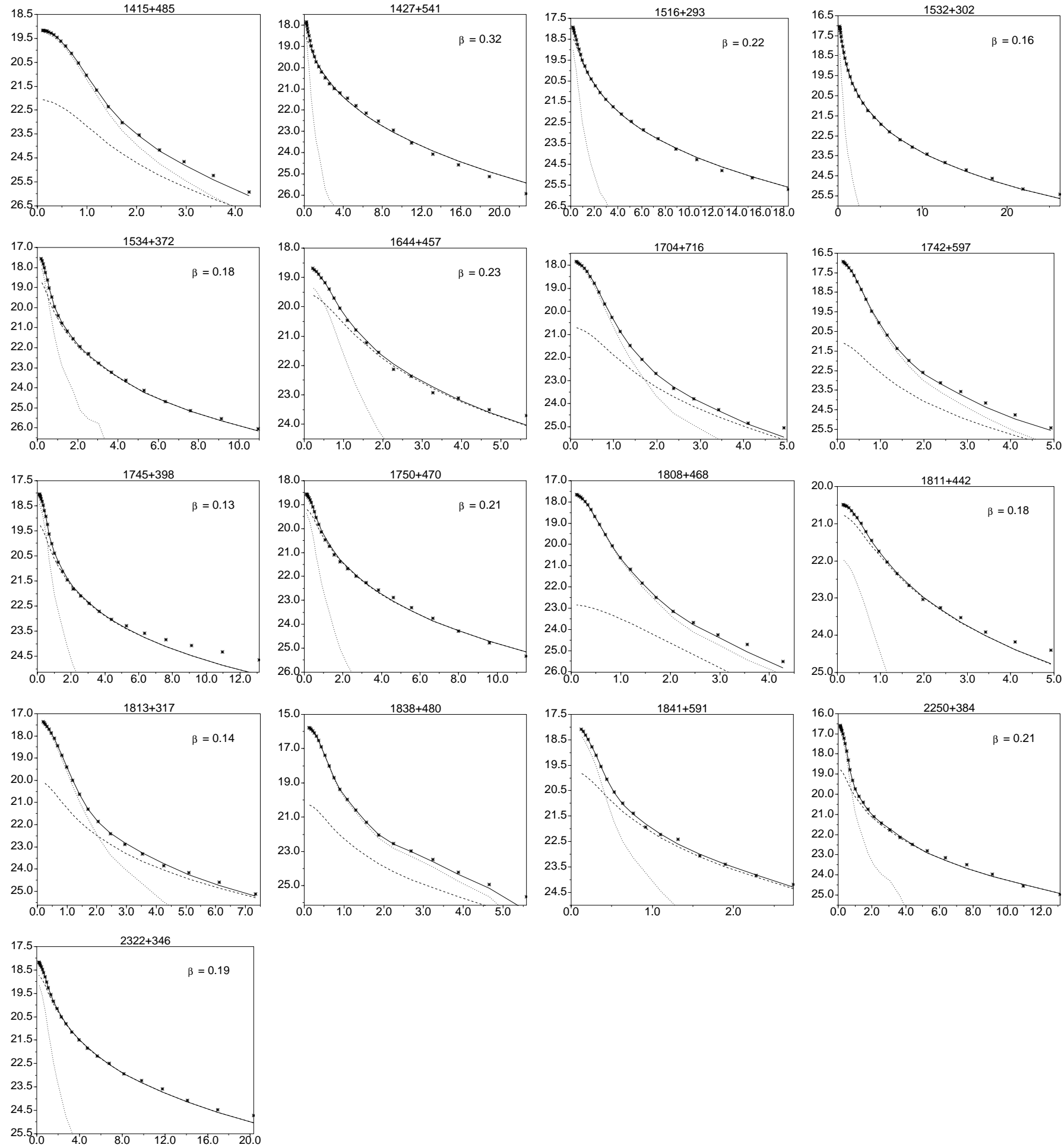

Fig. 2. Continued.

host galaxies do not always follow closely the de Vaucouleurs law. If one tries to fit a de Vaucouleurs model to a host galaxy that deviates from such law, different analysis methods may produce different results. We correlated the difference of $r_{\text {eff }}$ in Fig. 3b with $\beta$, but did not find an increasing difference of $r_{\text {eff }}$ with increasing deviation from $\beta=0.25$ as might be expected if the above effect were strong. It was also noted by P02, that the fitted effective radius depends on the outer radius of the fit region, which might contribute to the scatter.

\subsection{Notes on individual objects}

0007+472: This object is unresolved, but our upper limit for the host galaxy luminosity $\left(M_{R}>-22.4\right)$ indicates that the host galaxy should have been easily detected at $z=0.280$ (see also Fig. 4) . The redshift is from Kock et al. (1996), who report two possible absorption features $(G$-band and $\mathrm{NaD})$ at $z \sim 0.28$ in a "partially reduced spectrum". The redshift clearly needs to be checked. 

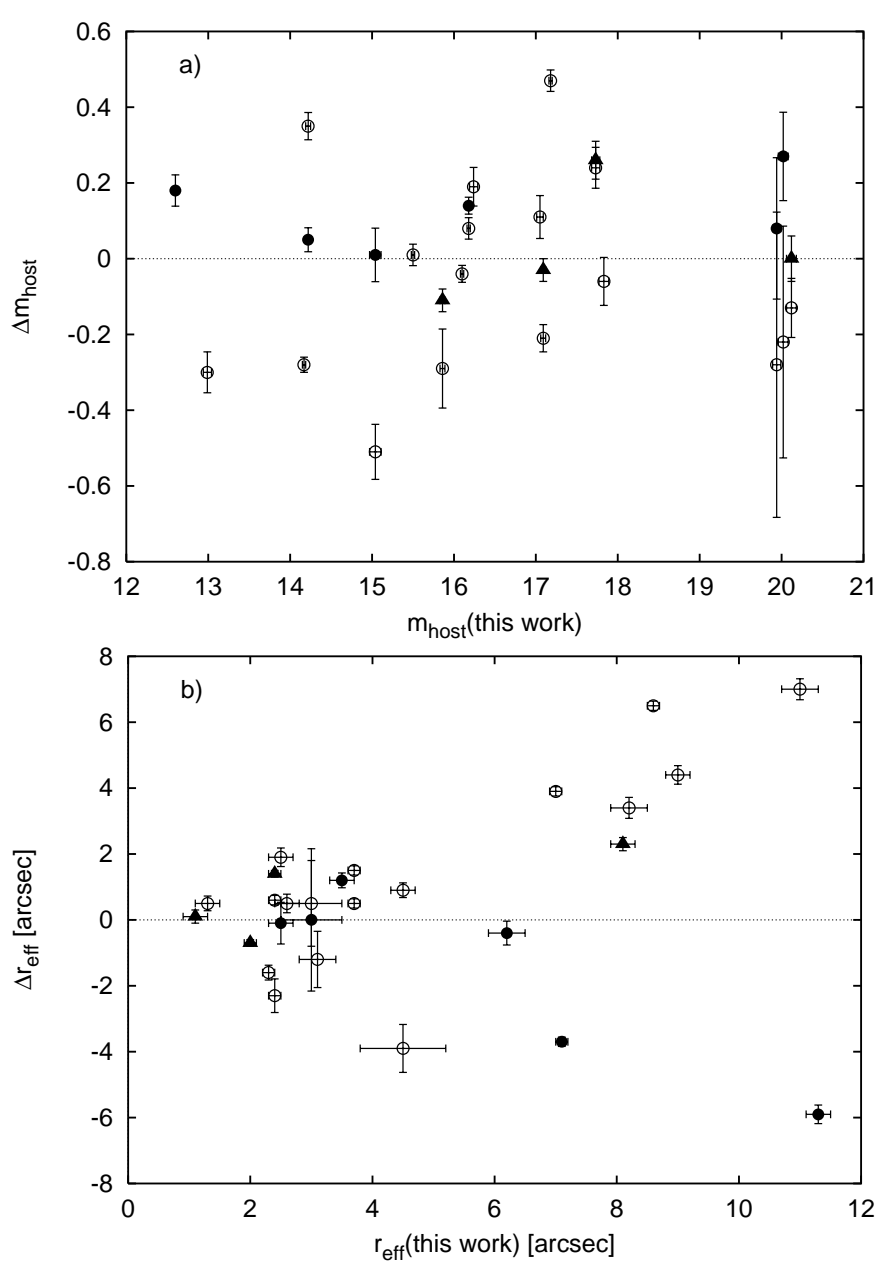

Fig. 3. The comparison of the fitted a) magnitude and b) effective radius with previous results (this work - comparison work). Filled triangles: Falomo \& Kotilainen (1999); open circles: Scarpa et al. (2000a); filled circles: Pursimo et al. (2002).

0035+598: This object consists of two point sources separated by $1.6 \operatorname{arcsec}$ (Scarpa et al. 1999a, FK99). Of these, the eastern object (object B in Scarpa et al. 1999a) is the likely BL Lac candidate. Gravitational lensing seems to be ruled out by the color difference of the two components. FK99 found the object resolved but noted the possibility of contaminating light from a nearby bright star. Our fit for this object was made by iterating between the object and the nearby companion and subtracting either of the objects before fitting the other. We find both objects to be unresolved and subtracting both of them leaves no significant residuals, except the faint galaxy (galaxy $\mathrm{G}$ in Scarpa et al. 1999a) 1.4 arcsec south of the BL Lac.

0040+408: This BL Lac candidate is located towards M 31 . The background is peppered with stars of M 31 and a correct determination of the background is impossible. Thus we did not attempt to fit a host galaxy model to this object. Subtraction of a point source reveals a faint object $\sim 0.9$ arcsec away at PA $-53^{\circ}$. It is not clear whether the nearby companion belongs to M 31 or is associated with the BL Lac object.

0109+182: Bauer et al. (2000) give $z=0.145$ for this object. A weak nebulosity is observed after subtracting the core (Fig. 10). This nebulosity corresponds to a host galaxy with
Table 2. Estimated redshifts for resolved objects without a spectroscopically determined redshift. See the text for details on the estimation.

\begin{tabular}{lccc}
\hline \hline Object & $R_{\text {host }}$ & $\begin{array}{c}r_{\text {eff }} \\
\text { [arcsec] }\end{array}$ & $z_{\text {est }}$ \\
\hline $0115+253$ & 18.63 & 2.9 & 0.35 \\
$0202+088$ & 19.90 & 2.9 & 0.55 \\
$0227+020$ & 19.56 & 1.6 & 0.45 \\
$0250+172$ & 17.00 & 5.7 & 0.25 \\
$0505+042$ & 18.05 & 3.8 & 0.35 \\
$0757+099$ & 18.55 & 1.3 & 0.30 \\
$0806+595$ & 17.14 & 5.8 & 0.30 \\
$1415+485$ & 20.09 & 1.2 & 0.50 \\
$1704+716$ & 19.06 & 1.6 & 0.35 \\
$1742+597$ & 19.33 & 1.6 & 0.40 \\
$1808+468$ & 19.97 & 1.2 & 0.45 \\
$1811+442$ & 18.24 & 3.6 & 0.35 \\
$1838+480$ & 19.12 & 1.0 & 0.30 \\
\hline
\end{tabular}

$R=18.75 \pm 0.11$ and $r_{\mathrm{eff}}=1.2 \pm 0.3$ arcsec. The error in the effective radius does not satisfy our criterion for a resolved object, thus this object is denoted unresolved in Table 4. With the above values the host galaxy would be rather faint $\left(M_{R}=-22.0\right)$ and small $\left(r_{\mathrm{eff}}=4 \mathrm{kpc}\right)$.

0250+172: Bauer et al. (2000) give $z=1.10$ for this BL Lac. The object is clearly resolved, and at $z=1.10$ the host galaxy would be exceedingly bright $\left(M_{R}<-29.0\right)$ and large $\left(r_{\mathrm{eff}}=\right.$ $64 \mathrm{kpc})$. Thus the redshift is most likely too high. There is a faint $(R=22.0)$ object 0.9 arcsec away from the nucleus at $\mathrm{PA} \sim 90^{\circ}$. We could not determine if this object is resolved or not, but it could be a distant star-forming galaxy giving rise to the high redshift in Bauer et al. (2000).

0314+247: A dust lane is visible crossing the host galaxy SE of the nucleus. A second, fainter dust lane is visible south of the nucleus. In the $\log \left(r_{\text {eff }}\right)-\mu_{\text {eff }}$ projection of the fundamental plane (Fig. 9) this object lies below the general trend, probably due to the absorption by dust in the host galaxy.

0326+024: Falomo (1996) noted the two nearby companions roughly west of the nucleus. The outer one seems to be severely distorted, possibly due to interaction with the BL Lac host galaxy. It could also consist of two overlapping galaxies.

0416+010: This BL Lac has been previously imaged extensively (see Table 3). The host galaxy parameters have been determined by 7 studies with the host magnitude and effective radius ranging from 17.45 to 17.80 and from 0.8 to 6.1 arcsec, respectively. Our values (17.73 and $2.9 \mathrm{arcsec})$ lie within this range.

0424+006: This object was found to be resolved by Abraham et al. (1991) and Falomo (1996) and unresolved by Wright et al. (1998b). We find the object unresolved, which may be due to the fact that the nucleus was very bright $(R=13.7)$ during our observations.

0505+072: Bauer et al. (2000) give $z=0.0272$, but they note that the spectrum is of poor quality. At this redshift the host galaxy would be very faint $\left(M_{R}=-18.3\right)$ and small $\left(r_{\mathrm{eff}}=\right.$ $3 \mathrm{kpc})$. The redshift probably needs to be checked. 
0650+250: As in all previous studies, this object remains unresolved. FK99 note that the tentative redshift 0.203 would lead to a relatively faint host galaxy.

0757+099: All previous authors find this object barely resolved. Falomo (1996) gives $R=18.9$ and $r_{\mathrm{eff}}=2.3 \mathrm{arcsec}$ for the host galaxy and SUO0 $R=19.2$ and $r_{\text {eff }}=2$ arcsec. Our results indicate a slightly brighter $(R=18.55)$ and smaller $\left(r_{\text {eff }}=1.3\right.$ arcsec $)$ host galaxy. This object has a tentative redshift $z=0.66$, but this would lead to a very bright host galaxy (SU00). The galaxy 14 arcsec NE of $0757+099$ has a redshift $z=0.270$ (Pesce et al. 1995). At this redshift our results would indicate $M_{R}=-23.2$, and $r_{\text {eff }}=7.2 \mathrm{kpc}$.

0814+296: Two faint objects are seen close to the object after subtracting the nucleus. The brighter one $(R \sim 22.0)$ is 2.2 arcsec away at $\mathrm{PA}-15^{\circ}$ and the fainter one $(R \sim 22.8)$ 1.3 arcsec away at PA $67^{\circ}$. No redshift information is available for the BL Lac or the nearby objects.

0854+441: A weak nebulosity is seen SE of the nucleus after point source subtraction. This nebulosity could be due to PSF subtraction errors.

0854+201: This object is one of the most studied in the sample, yet the host galaxy parameters are still poorly determined. SU00 found $0854+201$ to be unresolved. P02 found the object marginally resolved using the same dataset as in the present paper and a 1-dim. analysis method. The best-fitting host galaxy parameters corresponded to $R=18.9$ and $r_{\mathrm{eff}}=1.0$ arcsec. The object remains unresolved with the 2-dim. analysis method used in this study. We derive an upper limit $M_{R}>-24.0$ for the host galaxy. A weak nebulosity is seen around the nucleus after PSF subtraction extending a bit further towards $\mathrm{S}$ and SE. No redshift information is available for the close companion at 3.6 arcsec distance and PA $-21^{\circ}$. Our optical spectra obtained at the William Herschel Telescope (WHT) show that the diffuse galaxy 10 arcsec away at $\mathrm{PA}-15^{\circ}$ has a narrow emission line at $7200 \AA$ (Nilsson et al. 1995).

0915+295: Abraham et al. (1991) found the object unresolved. A faint nebulosity is seen SW of the nucleus after PSF subtraction (Fig. 11). We cannot determine if this nebulosity is due to an intervening galaxy or a nearby companion. The fits have been made under the assumption that it is a companion and the SW part of the object was masked out from the fit. However, an equally good fit can be achieved assuming a decentered host. In this case the host galaxy would have $R=16.02$ and $r_{\text {eff }}=1.5$ arcsec with a 0.2 arcsec decentering between the nucleus and the host. Higher resolution images are clearly needed to resolve the issue.

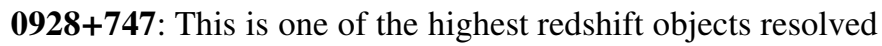
in this study $(z=0.638)$. Our results agree well with the those obtained with the HST (SU00).

1104+382: This object is discussed in more detail by Nilsson et al. (1999a). The bright companion galaxy was masked from the fit, but its outer parts may still be affecting the results. Gorham et al. (2000) find the companion to be a spiral with an unresolved nucleus.

1136+676: The host galaxy is clearly elongated towards a nearby galaxy, possibly due to interaction with it and the ellipticity of the host galaxy increases monotonically from 2 to 16 arcsec distance. Due to this increase our constant ellipticity model underestimates the flux at large distances from the nucleus (Fig. 2).

1136+701: The bright star $(R=15.16) 6.3 \operatorname{arcsec}$ away from the nucleus at PA $174^{\circ}$ was iteratively removed before final fitting.

1149+246: The redshift $(z=0.402)$ is from Beckmann et al. (2002, priv. comm.).

1217+301: The nearby companion galaxy SW of the nucleus may affect the fit results.

1221+282: There is a companion galaxy $(R \sim 18.0) \mathrm{SW}$ of the nucleus. The companion appears distorted and a weak bridge of emission extends towards the BL Lac nucleus from it. The bright nucleus made the determination of host galaxy parameters difficult, hence the large error in $\beta$.

1310+323: This is the highest redshift object in the sample $(z=0.996)$. We have a very deep exposure $(6000 \mathrm{~s})$ taken under good seeing conditions ( 0.9 arcsec), yet no sign of the host galaxy is seen. Urry et al. (1999) found the object also unresolved in their deep HST exposure. Stickel et al. (1993) noted that the outer isophotes are extended more to the west than to the east. There is a very faint galaxy $(R \sim 24.3)$ at $3.8 \operatorname{arcsec}$ distance and PA $-70^{\circ}$ that could be the cause of the isophote asymmetry.

1419+543: This is one of the two objects in the sample for which a formally better fit is obtained when using a disk model $(\beta=1.0)$ than when using the de Vaucouleurs model $(\beta=0.25)$. It has also the highest fitted $\beta$ (0.57) of the sample. Previous authors have alternatively described $1419+543$ as a disk galaxy (Abraham et al. 1991; Wurtz et al. 1996) or an elliptical galaxy (SU00, P02). Stickel et al. (1993) noted that the outer parts of the host ( $r>5$ arcsec) follow $\beta=0.25$ closely, whereas in the inner part there is an additional component that showed up after subtraction of the elliptical model. This object is discussed in more detail in Sect. 6.

1427+541: After subtracting the model a pattern resembling spiral arms is seen (Fig. 11). This BL Lac candidate could thus be hosted by a galaxy with a considerable disk component. The fitted $\beta$ is also among the highest (0.32) in the sample. However, the nucleus is very weak, and an almost equally good fit can be obtained without any nuclear component. The $4000 \AA$ break is also very close to the limit (39\%) set by LM99. It is therefore not clear if this object really has a BL Lac nucleus.

1442+541: This object was discussed in more detail in Heidt et al. (1999a). There is a very bright companion galaxy only 2.5 arcsec away whose physical association with $1442+120$ is confirmed by spectroscopy (Heidt, priv. comm.). Scarpa et al. (1999a) found a pointlike object 0.29 arcsec East of the BL Lac nucleus and discuss a possible lensing scenario. We do not detect this point source due to much lower resolution.

1516+293: This object has several bright companions that could not be masked out completely, and thus may affect the fit. 1517+654: Scarpa et al. (1999a) found three arclets and two bright spots surrounding the object, indicative of gravitational lensing. We barely detect the spots, and with a priori knowledge of their location also very faint trails of the arcs can be seen. The object remains unresolved as in FK99 and Scarpa et al. (1999a). 
Table 3. The observing log.

\begin{tabular}{|c|c|c|c|c|c|c|c|c|c|}
\hline RGB & Other name & $\mathrm{C}$ & $\mathrm{N}$ & $\overline{A_{R}}$ & $\begin{array}{l}\text { Date } \\
\mathrm{dd} / \mathrm{mm} / \mathrm{yy}\end{array}$ & Instr. $^{1}$ & $\begin{array}{r}\text { Exp. } \\
{[\mathrm{s}]}\end{array}$ & $\begin{array}{l}F W H M \\
{[\operatorname{arcsec}]}\end{array}$ & Prev. obs. \\
\hline $0007+472$ & RXJ 00079+4711 & \multirow{4}{*}{$\mathrm{y}$} & \multirow{6}{*}{$\mathrm{y}$} & 0.26 & $18 / 07 / 98$ & $\mathrm{~A}$ & 1200 & 1.1 & \multirow{6}{*}{$20,27,34,36$} \\
\hline $0035+598$ & 1ES $0033+595$ & & & 2.35 & $19 / 07 / 98$ & A & 900 & 1.1 & \\
\hline $0040+408$ & B3 $0037+405$ & & & 0.17 & $25 / 7 / 98$ & A & 1800 & 1.1 & \\
\hline $0109+182$ & RXJ 01090+1815 & & & 0.16 & $04 / 08 / 00$ & A & 1200 & 1.7 & \\
\hline $0110+418$ & RXJ 01100+4149 & $\mathrm{y}$ & & 0.19 & $04 / 07 / 97$ & A & 240 & 0.9 & \\
\hline & & & & & $02 / 09 / 98$ & A & & & \\
\hline $0112+227$ & S2 $0109+22$ & \multirow{4}{*}{$\mathrm{y}$} & & 0.10 & $15 / 12 / 98$ & A & 2070 & 1.2 & 10,18 \\
\hline $0115+253$ & RXJ 01157+2519 & & & 0.20 & $01 / 09 / 98$ & A & 720 & 0.8 & \\
\hline $0123+343$ & 1ES $0120+340$ & & & 0.16 & $18 / 01 / 02$ & A & 1200 & 1.0 & 20 \\
\hline $0136+391$ & B3 $0133+388$ & & $\mathrm{y}$ & 0.21 & $14 / 12 / 98$ & A & 1200 & 1.0 & \\
\hline $0152+017$ & PMNJ 0152+0146 & \multirow[t]{2}{*}{$\mathrm{y}$} & \multirow[t]{2}{*}{$\mathrm{y}$} & 0.09 & $14 / 12 / 98$ & A & 540 & 0.8 & \\
\hline $0153+712$ & $8 \mathrm{C} 0149+710$ & & & 1.63 & $01 / 09 / 98$ & A & 150 & 0.7 & \\
\hline $0202+088$ & PMNJ 0202+0848 & \multirow[t]{2}{*}{$\mathrm{y}$} & & 0.23 & $15 / 12 / 98$ & A & 1800 & 1.0 & \\
\hline $0214+517$ & RXJ 02142+5144 & & & 0.47 & $01 / 09 / 98$ & A & 150 & 0.8 & \\
\hline $0227+020$ & RXJ $02272+0201$ & \multirow[t]{2}{*}{$\mathrm{y}$} & & 0.08 & $15 / 12 / 98$ & A & 1800 & 1.2 & \\
\hline $0250+172$ & RXJ 02506+1712 & & $\mathrm{y}$ & 0.40 & $14 / 12 / 98$ & A & 1500 & 0.9 & \\
\hline $0314+247$ & RXJ 03140+2445 & \multirow[t]{9}{*}{$\mathrm{y}$} & \multirow[t]{2}{*}{$\mathrm{y}$} & 0.45 & $14 / 12 / 98$ & A & 1200 & 1.0 & \\
\hline $0316+090$ & RXJ 03161+0904 & & & 0.95 & $18 / 01 / 02$ & A & 900 & 1.1 & \\
\hline $0321+236$ & RXJ 03219+2336 & & \multirow[t]{4}{*}{$\mathrm{y}$} & 0.50 & $14 / 12 / 98$ & A & 1500 & 1.0 & \\
\hline $0326+024$ & $1 \mathrm{H} 0323+022$ & & & 0.30 & $14 / 12 / 98$ & A & 1600 & 1.1 & 10,11 \\
\hline $0416+010$ & $1 \mathrm{H} 0414+009$ & & & 0.32 & $15 / 12 / 98$ & A & 1200 & 0.9 & $1,3,4,10,11,20,23,34,36$ \\
\hline $0424+006$ & PKSJ 0424+0036 & & & 0.27 & $14 / 12 / 98$ & A & 660 & 1.1 & $2,10,18$ \\
\hline $0505+042$ & PMNJ 0505+0416 & & \multirow[t]{3}{*}{$\mathrm{y}$} & 0.21 & $14 / 12 / 98$ & A & 2400 & 1.0 & \\
\hline $0650+250$ & $1 \mathrm{ES} 0647+250$ & & & 0.26 & $14 / 12 / 98$ & A & 900 & 1.0 & $20,34,36$ \\
\hline $0654+427$ & B3 $0651+428$ & & & 0.26 & $14 / 12 / 98$ & A & 1500 & 1.0 & \\
\hline $0656+426$ & $4 C+42.22$ & \multirow[t]{9}{*}{$\mathrm{y}$} & $\mathrm{y}$ & 0.30 & $15 / 12 / 98$ & A & 600 & 0.9 & \\
\hline $0710+591$ & $1 \mathrm{H} 0658+595$ & & \multirow[t]{2}{*}{$\mathrm{y}$} & 0.10 & $14 / 12 / 98$ & A & 1200 & 1.0 & $30,34,35,36$ \\
\hline $0757+099$ & PKSJ 0757+0956 & & & 0.06 & $14 / 12 / 98$ & $\mathrm{~A}$ & 1600 & 1.0 & $2,10,18,34,36$ \\
\hline $0806+595$ & RXJ 08063+5931 & & \multirow[t]{6}{*}{$\mathrm{y}$} & 0.14 & $15 / 12 / 98$ & A & 1800 & 1.0 & \\
\hline $0814+296$ & EXO 0811.2+2949 & & & 0.10 & $15 / 12 / 98$ & A & 1800 & 0.9 & \\
\hline $0831+044$ & OJ 49 & & & 0.09 & $14 / 12 / 98$ & A & 1050 & 1.0 & $2,10,18,30,34,36$ \\
\hline $0854+441$ & B3 $0850+443$ & & & 0.07 & $15 / 12 / 98$ & A & 1800 & 1.0 & \\
\hline $0854+201^{2}$ & OJ 287 & & & 0.08 & $04 / 01 / 98$ & $\mathrm{~S}$ & 5160 & 0.6 & $7,9,11,16,19,23,34,35,36,37^{3}$ \\
\hline $0915+295$ & B2 $0912+29$ & & & 0.06 & $15 / 12 / 98$ & A & 1500 & 1.1 & 2 \\
\hline $0916+526$ & RXJ 09168+5238 & $\mathrm{y}$ & & 0.04 & $15 / 12 / 98$ & A & 1200 & 1.1 & \\
\hline $0928+747$ & $8 \mathrm{C} 0923+750$ & & & 0.09 & $15 / 12 / 98$ & A & 1800 & 0.9 & $11,20,34,36$ \\
\hline $0929+502$ & RXJ 09292+5013 & $\mathrm{y}$ & & 0.04 & $14 / 12 / 98$ & A & 1500 & 0.9 & \\
\hline $0930+350$ & $7 \mathrm{C} 0927+3516$ & & & 0.03 & $16 / 12 / 98$ & A & 1800 & 1.1 & \\
\hline $0952+656$ & RXJ 09524+6538 & & $\mathrm{y}$ & 0.37 & $14 / 12 / 98$ & A & 1800 & 0.9 & \\
\hline $1000+225 \mathrm{~A}$ & $4 \mathrm{C}+22.25$ & & & 0.08 & $15 / 12 / 98$ & A & 1800 & 1.0 & \\
\hline $1012+424$ & B3 $1009+427$ & $\mathrm{y}$ & $\mathrm{y}$ & 0.04 & $16 / 12 / 98$ & A & 1200 & 1.1 & \\
\hline $1015+494$ & $1 \mathrm{H} 1011+496$ & & & 0.03 & $16 / 12 / 98$ & A & 1200 & 1.0 & $20,34,36$ \\
\hline $1037+571$ & RXJ 10377+5711 & & & 0.01 & $16 / 12 / 98$ & A & 1500 & 0.9 & \\
\hline $1053+494$ & MS $1050.7+4946$ & & & 0.06 & $06 / 05 / 02$ & A & 600 & 1.0 & \\
\hline $1104+382^{2}$ & Mkn 421 & & & 0.04 & $05 / 01 / 98$ & $\mathrm{~S}$ & 600 & 0.5 & $11,25^{3}, 30,31,32,33,34,36$ \\
\hline $1136+676$ & RXJ 11365+6737 & & & 0.02 & $14 / 12 / 98$ & A & 1200 & 1.0 & \\
\hline $1136+701$ & Mkn 180 & & & 0.04 & $05 / 07 / 97$ & A & 90 & 1.2 & $2,30,34,36$ \\
\hline $1149+246$ & EXO 1149.9+2455 & & & 0.08 & $16 / 12 / 98$ & A & 1200 & 0.9 & \\
\hline $1151+589$ & $8 \mathrm{C} 1148+592$ & & & 0.08 & $05 / 07 / 97$ & A & 120 & 1.8 & \\
\hline $1217+301$ & 1ES $1215+303$ & & & 0.06 & $15 / 12 / 98$ & A & 600 & 1.1 & $2,34,36$ \\
\hline $1221+282$ & W Comae & & & 0.06 & $23 / 01 / 01$ & $\mathrm{~S}$ & 1280 & 0.6 & 11 \\
\hline $1230+253$ & RXJ 12302+2517 & & & 0.05 & $05 / 07 / 97$ & A & 120 & 1.4 & \\
\hline $1248+583$ & PG $1246+586$ & & & 0.03 & 05/07/97 & $\mathrm{A}$ & 120 & 1.2 & 34,36 \\
\hline $1310+323^{2}$ & AU CVn & & & 0.04 & $08 \& 11 / 07 / 96$ & $\mathrm{~S}$ & 6000 & 0.9 & $7,29,37^{3}$ \\
\hline $1341+399$ & RXJ 13410+3959 & $\mathrm{y}$ & & 0.02 & $06 / 05 / 02$ & A & 600 & 1.0 & \\
\hline $1415+485$ & RXJ $14155+4830$ & & $\mathrm{y}$ & 0.04 & $16 / 06 / 96$ & $\mathrm{H}$ & 600 & 1.2 & \\
\hline $1419+543^{2}$ & OQ 530 & & & 0.04 & $\begin{array}{l}08,11 \& \\
12 / 07 / 96\end{array}$ & $\mathrm{~S}$ & 6000 & 0.6 & $2,7,11,30,34,35,36,37^{3}$ \\
\hline
\end{tabular}


Table 3. Continued.

\begin{tabular}{|c|c|c|c|c|c|c|c|c|c|}
\hline RGB & Other name & $\overline{\mathrm{C}}$ & $\mathrm{N}$ & $\overline{A_{R}}$ & $\begin{array}{l}\text { Date } \\
\mathrm{dd} / \mathrm{mm} / \mathrm{yy}\end{array}$ & Instr. $^{1}$ & $\begin{array}{r}\text { Exp. } \\
{[\mathrm{s}]} \\
\end{array}$ & $\begin{array}{c}F W H M \\
{[\operatorname{arcsec}]}\end{array}$ & Prev. obs. \\
\hline $1427+541$ & RXJ 14274+5409 & $\mathrm{y}$ & $\mathrm{y}$ & 0.03 & $18 / 06 / 96$ & $S$ & 600 & 0.5 & \\
\hline $1428+426$ & $1 \mathrm{H} 1430+423$ & & & 0.03 & $19 / 06 / 96$ & $\mathrm{H}$ & 900 & 0.8 & $30,34,36$ \\
\hline $1439+395$ & PG $1437+398$ & & & 0.03 & $05 / 07 / 97$ & A & 120 & 1.2 & 34,36 \\
\hline $1442+120^{2}$ & 1ES $1440+122$ & & & 0.08 & $12 / 07 / 96$ & $S$ & 1200 & 0.6 & $21^{3}, 27,30,34,36$ \\
\hline $1448+361$ & RXJ 14479+3608 & & $\mathrm{y}$ & 0.03 & $17 / 06 / 96$ & $\mathrm{H}$ & 600 & 0.6 & \\
\hline $1454+514$ & RXJ 14544+5124 & $\mathrm{y}$ & $\mathrm{y}$ & 0.04 & $17 / 06 / 96$ & $\mathrm{H}$ & 600 & 0.7 & \\
\hline $1516+293$ & RXJ 15166+2917 & $\mathrm{y}$ & $\mathrm{y}$ & 0.07 & $18 / 06 / 96$ & $S$ & 900 & 0.6 & \\
\hline $1517+654 \mathrm{~B}$ & $1 \mathrm{H} 1515+660$ & & & 0.07 & $17 / 06 / 96$ & $\mathrm{H}$ & 600 & 0.8 & $20,27,34,36$ \\
\hline $1532+302$ & RXJ 15319+3016 & $\mathrm{y}$ & $\mathrm{y}$ & 0.08 & $18 / 06 / 96$ & $S$ & 900 & 0.5 & \\
\hline $1533+342$ & RXJ 15333+3416 & & $\mathrm{y}$ & 0.06 & $16 / 06 / 96$ & $\mathrm{H}$ & 600 & 0.7 & \\
\hline $1534+372$ & RXJ 15347+3716 & $\mathrm{y}$ & $\mathrm{y}$ & 0.05 & $18 / 06 / 96$ & $S$ & 600 & 0.6 & \\
\hline $1540+147^{2}$ & $4 \mathrm{C}+14.60$ & & & 0.15 & $09 / 07 / 96$ & $S$ & 4380 & 0.8 & $7,11,17,29,34,36,37^{3}$ \\
\hline $1554+201$ & MS $1552.1+2020$ & & & 0.13 & $04 / 07 / 97$ & A & 120 & 1.6 & 11,20 \\
\hline $1602+308$ & RXJ $16022+3050$ & & & 0.07 & $16 / 06 / 96$ & $\mathrm{H}$ & 600 & 0.6 & \\
\hline $1626+352$ & RXJ 16264+3513 & & & 0.06 & $01 / 09 / 98$ & A & 150 & 0.9 & \\
\hline $1644+457$ & B3 $1642+458$ & & & 0.04 & $31 / 08 / 98$ & A & 150 & 1.0 & \\
\hline $1652+403$ & RXJ $16527+4023$ & & $\mathrm{y}$ & 0.05 & $16 / 06 / 96$ & $\mathrm{H}$ & 600 & 0.6 & \\
\hline $1653+397^{2}$ & Mkn 501 & & & 0.05 & $08 \& 11 / 07 / 96$ & $S$ & 2670 & 0.7 & $2,6,7,8,11,25^{3}, 31,37$ \\
\hline $1704+716$ & RXJ 17047+7138 & & & 0.09 & $17 / 06 / 96$ & $\mathrm{H}$ & 600 & 0.8 & \\
\hline $1719+177$ & PKSJ 1719+1745 & & & 0.18 & $01 / 09 / 98$ & A & 360 & 0.9 & 2 \\
\hline $1728+502$ & $1 \mathrm{H} 1727+502$ & & & 0.08 & $06 / 07 / 97$ & A & 60 & 1.8 & $11,30,34,36$ \\
\hline $1742+597$ & RXJ 17424+5945 & & $\mathrm{y}$ & 0.12 & $17 / 06 / 96$ & $\mathrm{H}$ & 600 & 0.7 & \\
\hline $1743+195^{2}$ & 1ES $1741+196$ & & & 0.24 & $13 / 07 / 96$ & $S$ & 840 & 0.8 & $22^{3}$ \\
\hline $1745+398^{2}$ & B3 1743+398B & & $\mathrm{y}$ & 0.11 & $16 / 06 / 96$ & $\mathrm{H}$ & 1800 & 0.7 & $26^{3}$ \\
\hline $1748+700^{2}$ & S5 $1749+70$ & & & 0.08 & $21 / 10 / 95$ & $S$ & 5460 & 0.6 & $7,34,36,37^{3}$ \\
\hline $1749+433$ & B3 $1747+433$ & & & 0.09 & $31 / 08 / 98$ & A & 150 & 0.8 & \\
\hline $1750+470$ & B3 $1748+470$ & $\mathrm{y}$ & $\mathrm{y}$ & 0.09 & $17 / 06 / 96$ & $\mathrm{H}$ & 900 & 0.7 & \\
\hline $1756+553$ & RXJ 17561+5522 & & $\mathrm{y}$ & 0.10 & $17 / 06 / 96$ & $\mathrm{H}$ & 600 & 0.8 & \\
\hline $1806+698^{2}$ & $3 \mathrm{C} 371$ & & & 0.10 & $13 / 07 / 96$ & $S$ & 1530 & 0.6 & $7,11,15^{3}, 24,28,30,34,36,37$ \\
\hline $1808+468$ & RXJ 18079+4648 & & $\mathrm{y}$ & 0.10 & $17 / 06 / 96$ & $\mathrm{H}$ & 600 & 0.8 & \\
\hline $1811+442$ & RXJ 18118+4416 & & $\mathrm{y}$ & 0.21 & $17 / 06 / 96$ & $\mathrm{H}$ & 900 & 0.8 & \\
\hline $1813+317$ & EXO 1811.7+3143 & & & 0.13 & $19 / 07 / 98$ & A & 900 & 0.9 & \\
\hline $1824+568^{2}$ & $4 C+56.27$ & & & 0.16 & $11 / 07 / 96$ & $\mathrm{~S}$ & 2400 & 0.8 & $7,12,29,34,36,37^{3}$ \\
\hline $1829+540$ & RXJ 18293+5403 & & $\mathrm{y}$ & 0.09 & $17 / 06 / 96$ & $\mathrm{H}$ & 600 & 0.7 & \\
\hline $1838+480$ & RXJ 18387+4802 & & $\mathrm{y}$ & 0.15 & $19 / 06 / 96$ & $\mathrm{H}$ & 1200 & 0.6 & \\
\hline $1841+591$ & RXJ 18412+5906 & & $\mathrm{y}$ & 0.12 & $18 / 06 / 96$ & $\mathrm{~S}$ & 600 & 0.6 & \\
\hline $1848+427$ & RXJ 18487+4245 & & $\mathrm{y}$ & 0.21 & $17 / 06 / 96$ & $\mathrm{H}$ & 900 & 0.8 & \\
\hline $2039+523^{2}$ & 1ES $2037+521$ & $\mathrm{y}$ & & 2.44 & $11 / 07 / 96$ & $S$ & 1500 & 1.0 & $20,21^{3}, 34,36$ \\
\hline $2145+073$ & MS 2143.4+0704 & & & 0.20 & 05\&06/07/97 & A & 180 & 1.0 & $11,14,17,20,29,34,35,36$ \\
\hline $2202+422^{2}$ & BL Lacertae & & & 0.88 & $20 / 10 / 95$ & $S$ & 5400 & 0.8 & $7,11,30,34,36,37^{3}$ \\
\hline $2243+203$ & RXJ 22438+2020 & & $\mathrm{y}$ & 0.13 & $19 / 06 / 96$ & $\mathrm{H}$ & 300 & 0.6 & \\
\hline $2250+384$ & B3 $2247+381$ & $\mathrm{y}$ & $\mathrm{y}$ & 0.40 & $19 / 06 / 96$ & $\mathrm{H}$ & 600 & 0.6 & \\
\hline $2319+161$ & RXJ 23196+1611 & & $\mathrm{y}$ & 0.12 & $15 / 12 / 98$ & A & 800 & 1.2 & \\
\hline $2322+346$ & RXJ 23226+3436 & $\mathrm{y}$ & $\mathrm{y}$ & 0.32 & $17 / 07 / 98$ & A & 1200 & 1.1 & \\
\hline
\end{tabular}

${ }^{1} \mathrm{~A}=$ ALFOSC, $\mathrm{S}=$ StanCam, $\mathrm{H}=$ HiRAC.

${ }^{2}$ Data published before, reanalysis is made in this paper.

${ }^{3}$ Reference to the original publication.

References : 1. Falomo et al. (1990); 2. Abraham et al. (1991); 3. Falomo \& Tanzi (1991); 4. McHardy et al. (1992); 5. Romanishin (1992); 6. Kotilainen et al. (1993); 7. Stickel et al. (1993); 8. Kotilainen \& Ward (1994); 9. Benítez et al. (1996); 10. Falomo (1996); 11. Wurtz et al. (1996); 12. Falomo et al. (1997); 13. Gladders et al. (1997); 14. Jannuzi et al. (1997); 15. Nilsson et al. (1997); 16. Yanny et al. (1997); 17. Kotilainen et al. (1998); 18. Wright et al. (1998b); 19. Wright et al. (1998a); 20. Falomo \& Kotilainen (1999); 21. Heidt et al. (1999a); 22. Heidt et al. (1999b); 23. Heidt et al. (1999c); 24. Martel et al. (1999); 25. Nilsson et al. (1999a); 26. Nilsson et al. (1999b); 27. Scarpa et al. (1999a); 28. Scarpa et al. (1999b); 29. Urry et al. (1999); 30. Falomo et al. (2000); 31. Gonzáles-Serrano \& Carballo (2000); 32. Gorham et al. (2000); 33. Jansen et al. (2000); 34. Scarpa et al. (2000a); 35. Scarpa et al. (2000b); 36. Urry et al. (2000); 37. Pursimo et al. (2002). 
1540+147: Our result agrees well with the recent HST results for this high-redshift $(z=0.605)$ BL Lac. Urry et al. (1999) found $R=19.0$ and $r_{\mathrm{eff}}=2.4$ arcsec for the host galaxy and SU00 $R=20.2$ and $r_{\text {eff }}=2.5$ arcsec, whereas we find $R=19.9$ and $r_{\mathrm{eff}}=3.0$ arcsec. P02 found $R=19.9$ and $r_{\mathrm{eff}}=4.0 \mathrm{arcsec}$ using the same data as in this study. This object is one of the two objects for which an exponential host galaxy profile gives a better fit than a de Vaucouleurs profile. The $\chi^{2}$ is almost the same for both models, however. Urry et al. (1999) also found $1540+147$ equally well fitted by the two models

1626+352: The core plus a de Vaucouleurs fit for this object gives $R=19.73 \pm 0.12$ and $r_{\text {eff }}=0.8 \pm 0.2 \operatorname{arcsec}$ for the host galaxy. Because of the errors in $r_{\text {eff }}$ this object is denoted as unresolved in Table 4. At redshift $z=0.500$ the above values would indicate $M_{R}=-24.1$ and $r_{\text {eff }}=6 \mathrm{kpc}$.

1653+397: This object is discussed in more detail in Nilsson et al. (1999a). Fairly large residuals are seen in the inner part of the object after subtracting the best-fitting free $\beta$ model. Gonzáles-Serrano \& Carballo (2000) report 1653+397 to have disky isophotes.

1719+177: Abraham et al. (1991) found the object resolved, but could no determine the host galaxy type. We find the object unresolved, but our exposure time is relatively short (360 s).

1743+195: See Heidt et al. (1999b) for a detailed discussion of the object. The two nearby companions are at the same redshift as the BL Lac object.

1745+398: Nilsson et al. (1999b) discuss the arclike structure 8 arcsec SE of the BL Lac nucleus $(z=0.267)$ and show it to be a lensed image of a background galaxy at $z=1.057$. Assuming that the arc lies on the Einstein ring one obtains $M$ $1.3 \times 10^{13} M_{\odot}$ and $M / L_{\mathrm{B}} \sim 60$ inside a radius of $40 \mathrm{kpc}$. Like in $1136+676$, the ellipticity of the host galaxy increases outwards and our model underestimates the flux at large distances from the nucleus.

1748+700: This distant BL Lac $(z=0.770)$ was not resolved by the HST (SU00) and it remains unresolved in the deep NOT exposure (P02, this study).

1750+470: The bright nearby companion may be affecting the fit.

1806+698: Nilsson et al. (1997) discovered the optical jet roughly SW of the nucleus. This jet has also been imaged with HST (Martel et al. 1999; Scarpa et al. 1999b). There is a bridge of emission connecting the host galaxy of $1806+698$ to a pair of companion galaxies SW of the BL Lac. The radio structure of $1806+698$ is atypical, resembling more an FR II radio galaxy than an FR I radio galaxy (Wrobel \& Lind 1990).

1813+317: This object lies below the general trend in the $\mu_{\text {eff }}-r_{\text {eff }}$ plot (the object at $\log r_{\text {eff }} \sim 0.85$ and with larger errorbars in Fig. 9). There is no clear reason (bad PSF, bright companions, bad convergence of the fit) why the fit for $1813+371$ should be significantly worse than for the rest of the sample. The redshift (0.117) is from Giommi et al. (1991), and at this redshift the host galaxy would be rather faint $\left(M_{R}=-21.7\right)$ and small $\left(r_{\mathrm{eff}}=7 \mathrm{kpc}\right)$. The redshift of $1813+317$ could thus be actually higher than 0.117 , in which case it would move up and towards the right in Fig. 9.

1824+568: This is the highest redshift $(z=0.664)$ resolved object in the sample. We use the same data as P02 and our results agree well with each other $\left(R=19.75 \pm 0.10, r_{\mathrm{eff}}=\right.$ $2.6 \operatorname{arcsec}$ and $R=20.02 \pm 0.06, r_{\text {eff }}=2.5 \operatorname{arcsec}$ in $\mathrm{P} 02$ and this study, respectively). The two HST studies (Urry et al. 1999, SU00) find similar host magnitudes, but smaller host galaxy $(\sim 1$ arcsec).

1838+480: The nearby bright companion may be affecting the fit.

1848+427: The object remains unresolved, but faint emission is seen between the object and a nearby companion.

2039+523: The redshift $(z=0.053)$ is from our own observations at the Calar Alto $2.2 \mathrm{~m}$ telescope (Rector et al., in preparation). The nearby star was iteratively removed before final fitting. SU00 report a faint companion galaxy at 0.6 arcsec distance and PA $135^{\circ}$.

2202+422: The analysis of this object is complicated by the presence of numerous overlapping stars and galaxies, a nearby bright star, and diffuse galactic emission. Most of the Eastern side of the object was masked from the fit. There are higher than usual residuals in the inner part of the object, which could be due to an inner component or an artifact due to insufficient masking of the overlapping objects.

2319+161: Like $0035+598$ this object consists of two point sources close to each other (Fig. 11). An astrometric solution to 17 APM stars in the field pinpoints the radio coordinates to the NW object. The accuracy of this solution is $\sim 0.5$ arcsec. The nearby object is $3.3 \operatorname{arcsec}$ away at PA $124^{\circ}$. The spectra obtained at the Calar Alto $2.2 \mathrm{~m}$ telescope (Rector et al., in preparation) show the companion to be a galactic M class star.

\section{Discussion}

In this section we discuss briefly the general properties of the objects. A more detailed analysis will be given in a forthcoming paper.

In Fig. 4 we show the dependence of the host galaxy absolute magnitudes on redshift for the de Vaucouleurs model. Candidate objects are denoted with open symbols and confirmed BL Lacs with filled symbols. The solid line gives the behavior of an object with $R=19.0$ assuming $A_{R}=0.1$, the K-correction from Fukugita et al. (1995) and no evolution. The $R=19$ line seems to determine the bottom envelope of the data pretty well indicating an optical cutoff for the host galaxies at roughly this magnitude. As a result of this cutoff an increase of average absolute magnitude with redshift is seen.

At $z<0.2$, where the above selection effect is not yet serious, the candidate host galaxies appear slightly brighter $\left(M_{\text {host }}=-23.78 \pm 0.20\right)$ than the hosts of the confirmed BL Lacs $\left(M_{\text {host }}=-23.52 \pm 0.14\right)$, but using the K-S test the null hypothesis that both samples are drawn from the same distribution can be rejected at only $9 \%$ level. The average host absolute magnitude for the whole sample is -23.86 with a $0.75 \mathrm{mag}$ scatter which is in good agreement with recent studies, e.g. $-23.7 \pm 0.6$ (Urry et al. 2000) and $-23.85 \pm 0.59$ (FK99). The redshift distributions of the three samples (Fig. 5) differ slightly: the average redshift of resolved objects is lower in the present sample $(<z>=0.18)$ than the two comparison samples $(<z>=0.26$ and 0.24 in FK99 and Urry et al. 2000, respectively). However, none of the three studies detected host galaxies above $z=0.7$ 
Table 4. Results of the host galaxy fitting for the de Vaucouleurs model.

\begin{tabular}{|c|c|c|c|c|c|c|c|c|c|c|c|}
\hline RGB & $z$ & res? & $m_{\text {core }}$ & $m_{\text {host }}$ & $\begin{array}{c}r_{\mathrm{eff}} \\
\operatorname{arcsec}\end{array}$ & $\begin{array}{c}\mu_{\mathrm{eff}} \\
{\left[\mathrm{mag} / \mathrm{sq}^{\prime \prime}\right]}\end{array}$ & $\epsilon$ & PA & $\chi^{2}$ & $\frac{\chi_{\exp }^{2}}{\chi_{\text {deV }}^{2}}$ & $\begin{array}{l}\text { New } \\
\text { host? }\end{array}$ \\
\hline $0007+472$ & 0.280 & $\mathrm{U}$ & $18.69 \pm 0.03$ & $>19.6$ & - & - & - & - & 1.17 & - & - \\
\hline $0035+598$ & - & $\mathrm{U}$ & $18.39 \pm 0.05$ & - & - & - & - & - & 1.40 & - & - \\
\hline $0040+408$ & - & $\mathrm{U}$ & $19.43 \pm 0.05$ & - & - & - & - & - & 12.7 & - & - \\
\hline $0109+182$ & - & $\mathrm{U}$ & $16.44 \pm 0.02$ & - & - & - & - & - & 2.10 & - & - \\
\hline $0110+418$ & 0.096 & $\mathrm{R}$ & $18.62 \pm 0.05$ & $15.57 \pm 0.04$ & $6.6 \pm 0.1$ & $22.70 \pm 0.05$ & 0.29 & 62 & 0.99 & 1.72 & $\mathrm{Y}$ \\
\hline $0112+227$ & - & $\mathrm{U}$ & $14.96 \pm 0.05$ & - & - & - & - & - & 13.7 & - & - \\
\hline $0115+253$ & - & $\mathrm{R}$ & $18.02 \pm 0.04$ & $18.63 \pm 0.04$ & $2.9 \pm 0.2$ & $24.06 \pm 0.16$ & 0.22 & 173 & 1.17 & 1.12 & Y \\
\hline $0123+343$ & 0.272 & $\mathrm{R}$ & $17.98 \pm 0.03$ & $18.20 \pm 0.03$ & $1.7 \pm 0.1$ & $22.65 \pm 0.10$ & 0.08 & 138 & 1.05 & 1.19 & - \\
\hline $0136+391$ & - & $\mathrm{U}$ & $15.58 \pm 0.03$ & - & - & - & - & - & 4.58 & - & - \\
\hline $0152+017$ & 0.080 & $\mathrm{R}$ & $16.80 \pm 0.05$ & $14.65 \pm 0.05$ & $5.1 \pm 0.1$ & $21.40 \pm 0.05$ & 0.15 & 52 & 1.29 & 5.02 & $\mathrm{Y}$ \\
\hline $0153+712$ & 0.022 & $\mathrm{R}$ & $18.23 \pm 0.06$ & $13.33 \pm 0.05$ & $17.4 \pm 0.4$ & $22.87 \pm 0.06$ & 0.05 & 120 & 1.06 & 2.59 & $\mathrm{Y}$ \\
\hline $0202+088$ & - & $\mathrm{R}$ & $18.98 \pm 0.05$ & $19.90 \pm 0.09$ & $2.9 \pm 0.5$ & $25.45 \pm 0.32$ & 0.13 & 20 & 1.20 & 1.04 & $\mathrm{Y}$ \\
\hline $0214+517$ & 0.049 & $\mathrm{R}$ & $17.05 \pm 0.04$ & $14.54 \pm 0.04$ & $7.7 \pm 0.1$ & $22.18 \pm 0.04$ & 0.15 & 130 & 1.10 & 2.26 & $\mathrm{Y}$ \\
\hline $0227+020$ & - & $\mathrm{R}$ & $18.86 \pm 0.05$ & $19.56 \pm 0.06$ & $1.6 \pm 0.2$ & $23.85 \pm 0.22$ & 0.10 & 177 & 1.18 & 1.03 & Y \\
\hline $0250+172$ & - & $\mathrm{R}$ & $17.99 \pm 0.06$ & $17.00 \pm 0.06$ & $5.7 \pm 0.1$ & $23.86 \pm 0.06$ & 0.25 & 176 & 1.34 & 1.91 & $\mathrm{Y}$ \\
\hline $0314+247$ & 0.054 & $\mathrm{R}$ & $18.59 \pm 0.04$ & $16.15 \pm 0.04$ & $5.8 \pm 0.1$ & $23.01 \pm 0.04$ & 0.27 & 71 & 1.99 & 1.70 & Y \\
\hline $0316+090$ & - & $\mathrm{U}$ & $16.38 \pm 0.03$ & - & - & - & - & - & 3.03 & - & - \\
\hline $0321+236$ & - & $\mathrm{U}$ & $16.74 \pm 0.05$ & - & - & - & - & - & 13.2 & - & - \\
\hline $0326+024$ & 0.147 & $\mathrm{R}$ & $16.86 \pm 0.03$ & $17.05 \pm 0.03$ & $2.7 \pm 0.1$ & $22.33 \pm 0.10$ & 0.22 & 50 & 1.28 & 2.19 & - \\
\hline $0416+010$ & 0.287 & $\mathrm{R}$ & $17.18 \pm 0.05$ & $17.73 \pm 0.05$ & $2.9 \pm 0.1$ & $23.01 \pm 0.10$ & 0.32 & 80 & 1.18 & 1.51 & - \\
\hline $0424+006$ & - & $\mathrm{U}$ & $13.67 \pm 0.03$ & - & - & - & - & - & 12.9 & - & - \\
\hline $0505+042$ & - & $\mathrm{R}$ & $17.42 \pm 0.03$ & $18.05 \pm 0.04$ & $3.8 \pm 0.2$ & $24.03 \pm 0.12$ & 0.25 & 4 & 1.28 & 1.48 & $\mathrm{Y}$ \\
\hline $0650+250$ & - & $\mathrm{U}$ & $15.20 \pm 0.03$ & - & - & - & - & - & 5.38 & - & - \\
\hline $0654+427$ & 0.126 & $\mathrm{R}$ & $17.55 \pm 0.04$ & $15.98 \pm 0.04$ & $4.8 \pm 0.1$ & $22.65 \pm 0.05$ & 0.11 & 146 & 1.41 & 2.96 & $\mathrm{Y}$ \\
\hline $0656+426$ & 0.059 & $\mathrm{R}$ & $17.26 \pm 0.05$ & $13.99 \pm 0.05$ & $10.7 \pm 0.1$ & $22.20 \pm 0.05$ & 0.26 & 120 & 1.40 & 3.71 & Y \\
\hline $0710+591$ & 0.125 & $\mathrm{R}$ & $17.70 \pm 0.03$ & $15.81 \pm 0.03$ & $5.1 \pm 0.1$ & $22.44 \pm 0.04$ & 0.24 & 30 & 1.53 & 3.99 & - \\
\hline $0757+099$ & - & $\mathrm{R}$ & $16.32 \pm 0.03$ & $18.55 \pm 0.04$ & $1.3 \pm 0.2$ & $22.51 \pm 0.27$ & - & - & 1.81 & 1.22 & - \\
\hline $0806+595$ & - & $\mathrm{R}$ & $17.13 \pm 0.05$ & $17.14 \pm 0.05$ & $5.8 \pm 0.2$ & $24.10 \pm 0.07$ & 0.20 & 29 & 1.38 & 1.83 & $\mathrm{Y}$ \\
\hline $0814+296$ & - & $\mathrm{U}$ & $18.36 \pm 0.05$ & - & - & - & - & - & 6.36 & - & - \\
\hline $0831+044$ & 0.180 & $\mathrm{R}$ & $15.62 \pm 0.03$ & $17.05 \pm 0.04$ & $3.1 \pm 0.3$ & $22.44 \pm 0.20$ & 0.34 & 143 & 2.58 & 1.46 & - \\
\hline $0854+441$ & - & $\mathrm{U}$ & $17.05 \pm 0.05$ & - & - & - & - & - & 9.06 & - & - \\
\hline $0854+201$ & 0.306 & $\mathrm{U}$ & $15.33 \pm 0.05$ & $>18.1$ & - & - & - & - & 3.96 & - & - \\
\hline $0915+295$ & - & $\mathrm{U}$ & $15.88 \pm 0.05$ & - & - & - & - & - & 6.29 & - & - \\
\hline $0916+526$ & 0.190 & $\mathrm{R}$ & $18.42 \pm 0.05$ & $16.21 \pm 0.05$ & $5.0 \pm 0.1$ & $22.90 \pm 0.05$ & 0.16 & 17 & 1.11 & 2.55 & $\mathrm{Y}$ \\
\hline $0928+747$ & 0.638 & $\mathrm{R}$ & $20.25 \pm 0.07$ & $20.12 \pm 0.06$ & $1.3 \pm 0.2$ & $23.74 \pm 0.32$ & 0.27 & 73 & 1.00 & 1.07 & - \\
\hline $0929+502$ & - & $\mathrm{U}$ & $16.85 \pm 0.04$ & - & - & - & - & - & 5.09 & - & - \\
\hline $0930+350$ & - & $\mathrm{U}$ & $19.62 \pm 0.03$ & - & - & - & - & - & 1.45 & - & - \\
\hline $0952+656$ & - & $\mathrm{U}$ & $17.91 \pm 0.10$ & - & - & - & - & - & 1.81 & - & - \\
\hline $1000+225$ & 0.419 & $\mathrm{R}$ & $18.74 \pm 0.05$ & $18.63 \pm 0.05$ & $3.3 \pm 0.2$ & $24.42 \pm 0.11$ & 0.16 & 27 & 1.13 & 1.29 & $\mathrm{Y}$ \\
\hline $1012+424$ & 0.364 & $\mathrm{R}$ & $17.90 \pm 0.03$ & $18.13 \pm 0.04$ & $3.8 \pm 0.2$ & $24.36 \pm 0.11$ & 0.05 & 125 & 1.20 & 1.29 & $\mathrm{Y}$ \\
\hline $1015+494$ & 0.200 & $\mathrm{R}$ & $16.23 \pm 0.03$ & $17.09 \pm 0.03$ & $2.4 \pm 0.1$ & $22.04 \pm 0.13$ & 0.27 & 41 & 1.99 & 1.98 & - \\
\hline $1037+571$ & - & $\mathrm{U}$ & $15.99 \pm 0.03$ & - & - & - & - & - & 7.20 & - & - \\
\hline $1053+494$ & 0.140 & $\mathrm{R}$ & $17.79 \pm 0.07$ & $15.33 \pm 0.07$ & $7.1 \pm 0.1$ & $22.66 \pm 0.08$ & 0.25 & 69 & 1.09 & 1.69 & $\mathrm{Y}$ \\
\hline $1104+382$ & 0.031 & $\mathrm{R}$ & $12.91 \pm 0.05$ & $12.99 \pm 0.05$ & $11.0 \pm 0.3$ & $21.33 \pm 0.06$ & 0.21 & 105 & 6.02 & 2.66 & - \\
\hline $1136+676$ & 0.136 & $\mathrm{R}$ & $17.91 \pm 0.10$ & $16.10 \pm 0.10$ & $3.3 \pm 0.1$ & $21.93 \pm 0.10$ & 0.13 & 130 & 1.35 & 3.36 & $\mathrm{Y}$ \\
\hline $1136+701$ & 0.046 & $\mathrm{R}$ & $15.79 \pm 0.02$ & $14.17 \pm 0.02$ & $7.0 \pm 0.1$ & $21.73 \pm 0.03$ & 0.05 & 11 & 1.14 & 1.80 & - \\
\hline $1149+246$ & 0.402 & $\mathrm{R}$ & $19.28 \pm 0.04$ & $18.74 \pm 0.03$ & $1.1 \pm 0.1$ & $22.18 \pm 0.10$ & 0.13 & 158 & 1.20 & 1.42 & $\mathrm{Y}$ \\
\hline $1151+589$ & - & $\mathrm{U}$ & $16.51 \pm 0.02$ & - & - & - & - & - & 1.11 & - & - \\
\hline $1217+301$ & 0.130 & $\mathrm{R}$ & $14.84 \pm 0.05$ & $16.24 \pm 0.05$ & $4.5 \pm 0.7$ & $22.72 \pm 0.34$ & 0.15 & 18 & 2.43 & 1.44 & - \\
\hline $1221+282$ & 0.102 & $\mathrm{R}$ & $14.26 \pm 0.10$ & $16.60 \pm 0.10$ & $2.1 \pm 0.4$ & $21.36 \pm 0.40$ & 0.20 & 61 & 2.92 & 1.62 & - \\
\hline $1230+253$ & - & $\mathrm{U}$ & $15.45 \pm 0.02$ & - & - & - & - & - & 1.85 & - & - \\
\hline $1248+583$ & - & $\mathrm{U}$ & $15.74 \pm 0.02$ & - & - & - & - & - & 1.35 & - & - \\
\hline $1310+323$ & 0.996 & $\mathrm{U}$ & $17.92 \pm 0.02$ & $>20.6$ & - & - & - & - & 1.47 & - & - \\
\hline $1341+399$ & 0.163 & $\mathrm{R}$ & $18.36 \pm 0.07$ & $16.02 \pm 0.07$ & $6.8 \pm 0.2$ & $23.15 \pm 0.08$ & 0.32 & 84 & 1.07 & 1.77 & $\mathrm{Y}$ \\
\hline $1415+485$ & - & $\mathrm{R}$ & $18.51 \pm 0.04$ & $20.09 \pm 0.07$ & $1.2 \pm 0.2$ & $23.87 \pm 0.45$ & - & - & 1.08 & 1.00 & Y \\
\hline $1419+543$ & 0.151 & $\mathrm{R}$ & $15.51 \pm 0.02$ & $16.18 \pm 0.02$ & $4.5 \pm 0.2$ & $22.32 \pm 0.07$ & 0.38 & 146 & 3.58 & 0.94 & - \\
\hline $1427+541$ & 0.105 & $\mathrm{R}$ & $19.21 \pm 0.02$ & $14.95 \pm 0.02$ & $8.8 \pm 0.1$ & $22.91 \pm 0.02$ & 0.13 & 154 & 1.40 & 2.03 & Y \\
\hline
\end{tabular}


Table 4. Continued.

\begin{tabular}{|c|c|c|c|c|c|c|c|c|c|c|c|}
\hline RGB & $z$ & res? & $m_{\text {core }}$ & $m_{\text {host }}$ & $\begin{array}{c}r_{\mathrm{eff}} \\
\operatorname{arcsec}\end{array}$ & $\begin{array}{c}\mu_{\mathrm{eff}} \\
{\left[\mathrm{mag} / \mathrm{sq}^{\prime \prime}\right]}\end{array}$ & $\epsilon$ & PA & $\chi^{2}$ & $\frac{\chi_{\exp }^{2}}{\chi_{\mathrm{deV}}^{2}}$ & $\begin{array}{l}\text { New } \\
\text { host? }\end{array}$ \\
\hline $1428+426$ & 0.130 & $\mathrm{R}$ & $17.34 \pm 0.02$ & $16.10 \pm 0.02$ & $3.7 \pm 0.1$ & $21.83 \pm 0.03$ & 0.37 & 120 & 1.08 & 1.51 & - \\
\hline $1439+395$ & - & $\mathrm{U}$ & $16.36 \pm 0.02$ & - & - & - & - & - & 6.95 & - & - \\
\hline $1442+120$ & 0.162 & $\mathrm{R}$ & $17.56 \pm 0.02$ & $17.18 \pm 0.02$ & $2.3 \pm 0.1$ & $22.16 \pm 0.04$ & 0.18 & 170 & 1.60 & 2.30 & - \\
\hline $1448+361$ & - & $\mathrm{U}$ & $15.88 \pm 0.03$ & - & - & - & - & - & 2.02 & - & - \\
\hline $1454+514$ & - & $\mathrm{U}$ & $17.47 \pm 0.03$ & - & - & - & - & - & 1.22 & - & - \\
\hline $1516+293$ & 0.130 & $\mathrm{R}$ & $18.95 \pm 0.02$ & $15.73 \pm 0.02$ & $4.8 \pm 0.1$ & $22.36 \pm 0.03$ & 0.14 & 65 & 1.09 & 3.25 & Y \\
\hline $1517+654$ & - & $\mathrm{U}$ & $15.56 \pm 0.03$ & - & - & - & - & - & 1.97 & - & - \\
\hline $1532+302$ & 0.064 & $\mathrm{R}$ & $18.22 \pm 0.02$ & $15.01 \pm 0.02$ & $5.1 \pm 0.1$ & $21.89 \pm 0.02$ & 0.04 & 59 & 1.36 & 6.06 & Y \\
\hline $1533+342$ & - & $\mathrm{U}$ & $17.66 \pm 0.03$ & - & - & - & - & - & 1.20 & - & - \\
\hline $1534+372$ & 0.143 & $\mathrm{R}$ & $18.21 \pm 0.02$ & $17.18 \pm 0.02$ & $2.0 \pm 0.1$ & $21.98 \pm 0.04$ & 0.08 & 86 & 1.08 & 1.55 & $\mathrm{Y}$ \\
\hline $1540+147$ & 0.605 & $\mathrm{R}$ & $17.46 \pm 0.02$ & $19.94 \pm 0.05$ & $3.0 \pm 0.5$ & $25.71 \pm 0.32$ & - & - & 16.1 & 0.99 & - \\
\hline $1554+201$ & 0.222 & $\mathrm{R}$ & $18.03 \pm 0.06$ & $16.82 \pm 0.05$ & $2.8 \pm 0.1$ & $22.27 \pm 0.11$ & 0.15 & 148 & 1.09 & 1.06 & - \\
\hline $1602+308$ & - & $\mathrm{U}$ & $17.80 \pm 0.03$ & - & - & - & - & - & 1.11 & - & - \\
\hline $1626+352$ & 0.500 & $\mathrm{U}$ & $18.63 \pm 0.04$ & $>18.7$ & - & - & - & - & 1.18 & - & - \\
\hline $1644+457$ & 0.220 & $\mathrm{R}$ & $18.72 \pm 0.06$ & $17.04 \pm 0.06$ & $2.8 \pm 0.2$ & $22.53 \pm 0.13$ & 0.12 & 146 & 1.05 & 1.11 & $\mathrm{Y}$ \\
\hline $1652+403$ & - & $\mathrm{U}$ & $18.11 \pm 0.03$ & - & - & - & - & - & 1.20 & - & - \\
\hline $1653+397$ & 0.033 & $\mathrm{R}$ & $14.16 \pm 0.04$ & $12.60 \pm 0.04$ & $13.0 \pm 0.2$ & $21.26 \pm 0.04$ & 0.24 & 171 & 5.24 & 6.37 & - \\
\hline $1704+716$ & - & $\mathrm{R}$ & $17.77 \pm 0.03$ & $19.06 \pm 0.04$ & $1.6 \pm 0.1$ & $22.84 \pm 0.24$ & 0.44 & 18 & 1.10 & 1.01 & $\mathrm{Y}$ \\
\hline $1719+177$ & - & $\mathrm{U}$ & $17.86 \pm 0.04$ & - & - & - & - & - & 1.00 & - & - \\
\hline $1728+502$ & 0.055 & $\mathrm{R}$ & $16.56 \pm 0.03$ & $15.50 \pm 0.02$ & $3.7 \pm 0.1$ & $21.60 \pm 0.06$ & 0.11 & 38 & 1.15 & 1.10 & - \\
\hline $1742+597$ & - & $\mathrm{R}$ & $17.06 \pm 0.03$ & $19.33 \pm 0.06$ & $1.6 \pm 0.2$ & $23.74 \pm 0.38$ & - & - & 1.19 & 1.01 & Y \\
\hline $1743+195$ & 0.083 & $\mathrm{R}$ & $16.35 \pm 0.02$ & $14.47 \pm 0.03$ & $11.6 \pm 0.3$ & $22.71 \pm 0.04$ & 0.35 & 48 & 1.76 & 6.99 & - \\
\hline $1745+398$ & 0.267 & $\mathrm{R}$ & $18.38 \pm 0.03$ & $16.64 \pm 0.04$ & $6.0 \pm 0.1$ & $23.68 \pm 0.04$ & 0.20 & 159 & 1.27 & 2.02 & Y \\
\hline $1748+700$ & 0.770 & $\mathrm{U}$ & $16.07 \pm 0.05$ & $>18.7$ & - & - & - & - & 12.4 & - & - \\
\hline $1749+433$ & - & $\mathrm{U}$ & $18.89 \pm 0.04$ & - & - & - & - & - & 1.08 & - & - \\
\hline $1750+470$ & 0.160 & $\mathrm{R}$ & $19.30 \pm 0.04$ & $16.60 \pm 0.04$ & $4.4 \pm 0.1$ & $22.96 \pm 0.06$ & 0.20 & 5 & 1.14 & 1.60 & Y \\
\hline $1756+553$ & - & $\mathrm{U}$ & $17.02 \pm 0.03$ & - & - & - & - & - & 1.12 & - & - \\
\hline $1806+698$ & 0.051 & $\mathrm{R}$ & $14.89 \pm 0.03$ & $14.22 \pm 0.03$ & $8.6 \pm 0.1$ & $22.18 \pm 0.04$ & 0.09 & 64 & 1.97 & 4.87 & - \\
\hline $1808+468$ & - & $\mathrm{R}$ & $17.61 \pm 0.03$ & $19.97 \pm 0.08$ & $1.2 \pm 0.2$ & $23.75 \pm 0.59$ & - & - & 1.13 & 1.01 & Y \\
\hline $1811+442$ & - & $\mathrm{R}$ & $21.19 \pm 0.06$ & $18.24 \pm 0.04$ & $3.6 \pm 0.1$ & $24.18 \pm 0.07$ & 0.19 & 10 & 1.10 & 1.18 & $\mathrm{Y}$ \\
\hline $1813+317$ & 0.117 & $\mathrm{R}$ & $16.89 \pm 0.05$ & $17.92 \pm 0.05$ & $2.9 \pm 0.3$ & $23.26 \pm 0.22$ & 0.28 & 0 & 1.27 & 1.12 & $\mathrm{Y}$ \\
\hline $1824+568$ & 0.664 & $\mathrm{R}$ & $18.07 \pm 0.04$ & $20.02 \pm 0.06$ & $2.5 \pm 0.2$ & $25.40 \pm 0.19$ & - & - & 1.15 & 1.05 & - \\
\hline $1829+540$ & - & $\mathrm{U}$ & $17.81 \pm 0.03$ & - & - & - & - & - & 1.06 & - & - \\
\hline $1838+480$ & - & $\mathrm{R}$ & $16.29 \pm 0.02$ & $19.12 \pm 0.05$ & $1.0 \pm 0.1$ & $22.51 \pm 0.33$ & - & - & 1.62 & 1.04 & $\mathrm{Y}$ \\
\hline $1841+591$ & 0.530 & $\mathrm{R}$ & $19.63 \pm 0.02$ & $19.04 \pm 0.03$ & $2.1 \pm 0.1$ & $23.77 \pm 0.13$ & 0.22 & 82 & 1.10 & 1.07 & $\mathrm{Y}$ \\
\hline $1848+427$ & - & $\mathrm{U}$ & $18.75 \pm 0.03$ & - & - & - & - & - & 1.12 & - & - \\
\hline $2039+523$ & 0.053 & $\mathrm{R}$ & $19.30 \pm 0.03$ & $15.86 \pm 0.03$ & $9.0 \pm 0.2$ & $23.79 \pm 0.04$ & 0.19 & 120 & 1.17 & 3.49 & - \\
\hline $2145+073$ & 0.237 & $\mathrm{R}$ & $18.18 \pm 0.06$ & $17.83 \pm 0.06$ & $2.6 \pm 0.2$ & $22.91 \pm 0.14$ & 0.30 & 54 & 1.02 & 1.06 & - \\
\hline $2202+422$ & 0.069 & $\mathrm{R}$ & $14.93 \pm 0.07$ & $15.04 \pm 0.07$ & $8.2 \pm 0.3$ & $22.39 \pm 0.09$ & 0.43 & 33 & 4.88 & 8.59 & - \\
\hline $2243+203$ & - & $\mathrm{U}$ & $15.77 \pm 0.02$ & - & - & - & - & - & 3.14 & - & - \\
\hline $2250+384$ & 0.119 & $\mathrm{R}$ & $17.14 \pm 0.02$ & $16.04 \pm 0.03$ & $5.7 \pm 0.2$ & $23.06 \pm 0.05$ & 0.13 & 160 & 1.08 & 1.61 & $\mathrm{Y}$ \\
\hline $2319+161$ & - & $\mathrm{U}$ & $17.73 \pm 0.05$ & - & - & - & - & - & 1.10 & - & - \\
\hline $2322+346$ & 0.098 & $\mathrm{R}$ & $17.99 \pm 0.05$ & $15.30 \pm 0.05$ & $6.7 \pm 0.1$ & $22.51 \pm 0.06$ & 0.25 & 65 & 1.03 & 1.94 & $\mathrm{Y}$ \\
\hline
\end{tabular}

and all of them detected only a handful of hosts above $z=0.4$, so one would not expect large differences of average $M_{\text {host }}$ between the samples. The effective radii are also very similar to what has been found previously. The average effective radius of the RGB hosts is $13.2 \pm 0.8 \mathrm{kpc}$ for the de Vaucouleurs model and $19.9 \pm 2.0 \mathrm{kpc}$ for the free $\beta$ model.

At $z<0.2$ the nuclei of the BL Lac candidates seem to have systematically fainter nuclei than the confirmed $\mathrm{BL}$ Lacs. The average nuclear brightness is $-21.05 \pm 0.26$ for the candidate objects compared to $-22.54 \pm 0.32$ of the certain BL Lacs. This difference is not surprising since the candidate objects have mostly $25 \%<\mathrm{Br}_{4000 \AA}<40 \%$ indicative of weak nuclear activity and/or low nucleus/host galaxy flux ratios. The candidate nuclei are also fainter than the nuclei of the $1 \mathrm{Jy} \mathrm{BL}$ Lacs studied in P02 that are all brighter than $M_{R}=-22.0$. However, in the EMSS sample studied by FK99, many nuclei have $-22<M_{R}<-20$, i.e. the candidate nuclei are not unprecedentedly faint. The nuclear brightnesses of the candidates and certain BL Lacs seem to form a homogeneous distribution with no gap between them. This further supports the view that the candidates represent the low-luminosity end of the RGB BL Lacs.

As Table 4 shows, our model fits favor almost exclusively the de Vaucouleurs model $(\beta=0.25)$ over the exponential disk 
Table 5. Results of the host galaxy fitting for the free $\beta$ model.

\begin{tabular}{|c|c|c|c|c|c|c|c|c|c|}
\hline RGB & $m_{\text {core }}$ & $m_{\text {host }}$ & $\begin{array}{c}r_{\mathrm{eff}} \\
\operatorname{arcsec}\end{array}$ & $\begin{array}{c}\mu_{\text {eff }} \\
{\left[\mathrm{mag} / \mathrm{sq}^{\prime \prime}\right]}\end{array}$ & $\overline{\epsilon \epsilon}$ & PA & $\bar{\beta}$ & $\overline{\chi^{2}}$ & $\begin{array}{l}\text { New } \\
\text { host? }\end{array}$ \\
\hline $0110+418$ & $18.86 \pm 0.05$ & $15.48 \pm 0.05$ & $7.6 \pm 0.2$ & $22.99 \pm 0.06$ & 0.29 & 62 & $0.21 \pm 0.01$ & 0.98 & $\bar{Y}$ \\
\hline $0115+253$ & $18.05 \pm 0.04$ & $18.36 \pm 0.11$ & $4.2 \pm 0.5$ & $24.83 \pm 0.22$ & 0.22 & 173 & $0.16 \pm 0.03$ & 1.17 & $\mathrm{Y}$ \\
\hline $0123+343$ & $18.10 \pm 0.04$ & $17.88 \pm 0.07$ & $2.0 \pm 0.2$ & $23.07 \pm 0.16$ & 0.08 & 137 & $0.12 \pm 0.02$ & 1.04 & - \\
\hline $0152+017$ & $16.92 \pm 0.05$ & $14.56 \pm 0.05$ & $5.7 \pm 0.1$ & $21.64 \pm 0.05$ & 0.15 & 51 & $0.21 \pm 0.01$ & 1.24 & $\mathrm{Y}$ \\
\hline $0153+712$ & $19.28 \pm 0.09$ & $13.03 \pm 0.07$ & $29.1 \pm 1.8$ & $23.85 \pm 0.13$ & 0.05 & 119 & $0.18 \pm 0.01$ & 1.01 & $\mathrm{Y}$ \\
\hline $0202+088$ & $18.98 \pm 0.05$ & $19.83 \pm 0.19$ & $3.3 \pm 1.2$ & $25.73 \pm 0.53$ & 0.13 & 19 & $0.22 \pm 0.07$ & 1.20 & Y \\
\hline $0214+517$ & $17.58 \pm 0.06$ & $14.08 \pm 0.06$ & $17.4 \pm 1.0$ & $23.88 \pm 0.15$ & 0.15 & 132 & $0.12 \pm 0.01$ & 1.00 & $\mathrm{Y}$ \\
\hline $0227+020$ & $18.86 \pm 0.06$ & $19.58 \pm 0.12$ & $1.6 \pm 0.2$ & $23.85 \pm 0.23$ & 0.10 & 176 & $0.26 \pm 0.06$ & 1.18 & Y \\
\hline $0250+172$ & $17.98 \pm 0.06$ & $17.02 \pm 0.06$ & $5.6 \pm 0.3$ & $23.82 \pm 0.12$ & 0.25 & 176 & $0.26 \pm 0.01$ & 1.34 & Y \\
\hline $0314+247$ & $18.36 \pm 0.04$ & $16.39 \pm 0.04$ & $4.2 \pm 0.1$ & $22.34 \pm 0.05$ & 0.25 & 71 & $0.40 \pm 0.01$ & 1.79 & Y \\
\hline $0326+024$ & $17.01 \pm 0.04$ & $16.60 \pm 0.07$ & $3.9 \pm 0.3$ & $23.17 \pm 0.18$ & 0.21 & 50 & $0.10 \pm 0.01$ & 1.15 & - \\
\hline $0416+010$ & $17.25 \pm 0.05$ & $17.39 \pm 0.09$ & $4.2 \pm 0.3$ & $23.85 \pm 0.19$ & 0.33 & 80 & $0.12 \pm 0.02$ & 1.15 & - \\
\hline $0505+042$ & $17.45 \pm 0.03$ & $17.65 \pm 0.06$ & $7.1 \pm 0.5$ & $25.31 \pm 0.16$ & 0.26 & 4 & $0.13 \pm 0.01$ & 1.22 & $\mathrm{Y}$ \\
\hline $0654+427$ & $17.91 \pm 0.05$ & $15.50 \pm 0.06$ & $10.3 \pm 0.7$ & $24.25 \pm 0.17$ & 0.12 & 146 & $0.11 \pm 0.01$ & 1.16 & Y \\
\hline $0656+426$ & $18.58 \pm 0.08$ & $13.56 \pm 0.05$ & $22.9 \pm 0.7$ & $23.75 \pm 0.08$ & 0.27 & 119 & $0.13 \pm 0.01$ & 1.10 & $\mathrm{Y}$ \\
\hline $0710+591$ & $18.09 \pm 0.05$ & $15.46 \pm 0.04$ & $8.4 \pm 0.4$ & $23.52 \pm 0.10$ & 0.24 & 31 & $0.13 \pm 0.01$ & 1.28 & - \\
\hline $0806+595$ & $17.17 \pm 0.05$ & $16.74 \pm 0.06$ & $11.2 \pm 0.6$ & $25.44 \pm 0.13$ & 0.20 & 29 & $0.14 \pm 0.01$ & 1.33 & $\mathrm{Y}$ \\
\hline $0831+044$ & $15.68 \pm 0.04$ & $16.37 \pm 0.09$ & $6.5 \pm 1.0$ & $24.16 \pm 0.38$ & 0.33 & 143 & $0.06 \pm 0.01$ & 2.34 & - \\
\hline $0916+526$ & $18.63 \pm 0.06$ & $16.03 \pm 0.05$ & $6.5 \pm 0.2$ & $23.48 \pm 0.08$ & 0.15 & 17 & $0.18 \pm 0.01$ & 1.07 & Y \\
\hline $0928+747$ & $20.30 \pm 0.09$ & $19.99 \pm 0.10$ & $1.4 \pm 0.2$ & $23.90 \pm 0.32$ & 0.28 & 73 & $0.19 \pm 0.04$ & 1.00 & - \\
\hline $1000+225$ & $18.82 \pm 0.05$ & $18.25 \pm 0.09$ & $5.8 \pm 0.5$ & $25.61 \pm 0.21$ & 0.16 & 28 & $0.13 \pm 0.02$ & 1.11 & Y \\
\hline $1012+424$ & $17.96 \pm 0.03$ & $17.64 \pm 0.09$ & $8.6 \pm 1.1$ & $26.08 \pm 0.33$ & 0.05 & 129 & $0.11 \pm 0.02$ & 1.15 & Y \\
\hline $1015+494$ & $16.37 \pm 0.04$ & $16.40 \pm 0.08$ & $4.0 \pm 0.5$ & $23.21 \pm 0.33$ & 0.28 & 42 & $0.06 \pm 0.01$ & 1.63 & - \\
\hline $1053+494$ & $18.28 \pm 0.09$ & $14.98 \pm 0.08$ & $12.4 \pm 0.7$ & $23.83 \pm 0.14$ & 0.25 & 69 & $0.14 \pm 0.01$ & 1.04 & $\mathrm{Y}$ \\
\hline $1104+382$ & $12.89 \pm 0.05$ & $13.18 \pm 0.05$ & $8.2 \pm 0.1$ & $20.69 \pm 0.06$ & 0.21 & 105 & $0.36 \pm 0.02$ & 5.61 & - \\
\hline $1136+676$ & $18.21 \pm 0.11$ & $15.89 \pm 0.10$ & $4.1 \pm 0.1$ & $22.43 \pm 0.12$ & 0.13 & 130 & $0.16 \pm 0.01$ & 1.24 & $\mathrm{Y}$ \\
\hline $1136+701$ & $15.90 \pm 0.03$ & $14.04 \pm 0.03$ & $8.3 \pm 0.3$ & $22.12 \pm 0.07$ & 0.04 & 15 & $0.19 \pm 0.01$ & 1.12 & - \\
\hline $1149+246$ & $19.52 \pm 0.05$ & $18.51 \pm 0.05$ & $1.1 \pm 0.1$ & $22.41 \pm 0.13$ & 0.11 & 159 & $0.11 \pm 0.01$ & 1.11 & $\mathrm{Y}$ \\
\hline $1217+301$ & $14.94 \pm 0.06$ & $15.34 \pm 0.31$ & $19.4 \pm 6.2$ & $26.04 \pm 1.02$ & 0.11 & 13 & $0.04 \pm 0.06$ & 1.92 & - \\
\hline $1221+282$ & $14.29 \pm 0.10$ & $16.02 \pm 0.22$ & $3.4 \pm 0.4$ & $22.42 \pm 0.26$ & 0.21 & 59 & $0.08 \pm 0.06$ & 2.60 & - \\
\hline $1341+399$ & $18.47 \pm 0.07$ & $15.88 \pm 0.08$ & $8.4 \pm 0.4$ & $23.59 \pm 0.12$ & 0.32 & 84 & $0.20 \pm 0.01$ & 1.06 & Y \\
\hline $1419+543$ & $15.47 \pm 0.02$ & $16.51 \pm 0.02$ & $3.7 \pm 0.1$ & $21.80 \pm 0.02$ & 0.38 & 146 & $0.57 \pm 0.04$ & 2.31 & - \\
\hline $1427+541$ & $18.91 \pm 0.02$ & $15.11 \pm 0.03$ & $6.8 \pm 0.2$ & $22.40 \pm 0.06$ & 0.12 & 154 & $0.32 \pm 0.01$ & 1.36 & $\mathrm{Y}$ \\
\hline $1428+426$ & $17.37 \pm 0.02$ & $16.06 \pm 0.03$ & $3.8 \pm 0.1$ & $21.89 \pm 0.05$ & 0.37 & 120 & $0.23 \pm 0.01$ & 1.08 & - \\
\hline $1442+120$ & $17.59 \pm 0.02$ & $17.09 \pm 0.03$ & $2.6 \pm 0.1$ & $22.43 \pm 0.04$ & 0.18 & 171 & $0.21 \pm 0.01$ & 1.59 & - \\
\hline $1516+293$ & $19.11 \pm 0.04$ & $15.66 \pm 0.03$ & $5.3 \pm 0.2$ & $22.57 \pm 0.08$ & 0.14 & 65 & $0.22 \pm 0.01$ & 1.08 & $\mathrm{Y}$ \\
\hline $1532+302$ & $18.87 \pm 0.04$ & $14.75 \pm 0.02$ & $7.8 \pm 0.3$ & $22.79 \pm 0.08$ & 0.04 & 60 & $0.16 \pm 0.01$ & 1.11 & $\mathrm{Y}$ \\
\hline $1534+372$ & $18.33 \pm 0.02$ & $17.04 \pm 0.03$ & $2.2 \pm 0.1$ & $22.22 \pm 0.08$ & 0.08 & 87 & $0.18 \pm 0.01$ & 1.07 & Y \\
\hline $1554+201$ & $18.03 \pm 0.08$ & $16.83 \pm 0.08$ & $2.8 \pm 0.1$ & $22.28 \pm 0.11$ & 0.15 & 148 & $0.25 \pm 0.04$ & 1.09 & - \\
\hline $1644+457$ & $18.74 \pm 0.08$ & $17.01 \pm 0.08$ & $2.9 \pm 0.2$ & $22.63 \pm 0.18$ & 0.11 & 147 & $0.23 \pm 0.03$ & 1.05 & Y \\
\hline $1653+397$ & $14.45 \pm 0.04$ & $11.94 \pm 0.06$ & $45.0 \pm 4.2$ & $23.78 \pm 0.20$ & 0.24 & 171 & $0.10 \pm 0.01$ & 1.50 & - \\
\hline $1704+716$ & $17.75 \pm 0.04$ & $19.21 \pm 0.13$ & $1.6 \pm 0.1$ & $22.82 \pm 0.18$ & 0.43 & 17 & $0.36 \pm 0.12$ & 1.10 & Y \\
\hline $1728+502$ & $16.62 \pm 0.04$ & $15.46 \pm 0.04$ & $3.8 \pm 0.1$ & $21.69 \pm 0.06$ & 0.11 & 38 & $0.22 \pm 0.02$ & 1.15 & - \\
\hline $1743+195$ & $16.49 \pm 0.02$ & $14.07 \pm 0.06$ & $23.4 \pm 2.2$ & $24.11 \pm 0.18$ & 0.35 & 48 & $0.15 \pm 0.01$ & 1.32 & - \\
\hline $1745+398$ & $18.56 \pm 0.03$ & $16.14 \pm 0.06$ & $14.4 \pm 1.0$ & $25.41 \pm 0.16$ & 0.21 & 158 & $0.13 \pm 0.01$ & 1.17 & Y \\
\hline $1750+470$ & $19.45 \pm 0.04$ & $16.51 \pm 0.05$ & $5.1 \pm 0.3$ & $23.28 \pm 0.12$ & 0.20 & 4 & $0.21 \pm 0.01$ & 1.13 & Y \\
\hline $1806+698$ & $15.00 \pm 0.03$ & $13.68 \pm 0.05$ & $22.0 \pm 1.6$ & $24.11 \pm 0.16$ & 0.09 & 63 & $0.11 \pm 0.01$ & 1.29 & - \\
\hline $1811+442$ & $21.65 \pm 0.16$ & $18.02 \pm 0.07$ & $5.0 \pm 0.5$ & $24.85 \pm 0.19$ & 0.19 & 11 & $0.18 \pm 0.02$ & 1.08 & Y \\
\hline $1813+317$ & $16.92 \pm 0.05$ & $17.63 \pm 0.11$ & $4.0 \pm 0.5$ & $23.96 \pm 0.27$ & 0.29 & 0 & $0.14 \pm 0.03$ & 1.26 & $\mathrm{Y}$ \\
\hline $1841+591$ & $19.61 \pm 0.03$ & $19.11 \pm 0.10$ & $2.0 \pm 0.2$ & $23.66 \pm 0.23$ & 0.22 & 82 & $0.29 \pm 0.06$ & 1.10 & Y \\
\hline $2039+523$ & $19.55 \pm 0.03$ & $15.65 \pm 0.04$ & $12.5 \pm 0.7$ & $24.40 \pm 0.10$ & 0.20 & 120 & $0.20 \pm 0.01$ & 1.11 & - \\
\hline $2145+073$ & $18.19 \pm 0.07$ & $17.82 \pm 0.10$ & $2.6 \pm 0.2$ & $22.92 \pm 0.19$ & 0.30 & 54 & $0.24 \pm 0.04$ & 1.02 & - \\
\hline $2202+422$ & $14.95 \pm 0.07$ & $14.88 \pm 0.09$ & $10.4 \pm 0.8$ & $22.89 \pm 0.08$ & 0.43 & 34 & $0.19 \pm 0.02$ & 4.40 & - \\
\hline $2250+384$ & $17.17 \pm 0.02$ & $15.92 \pm 0.05$ & $6.8 \pm 0.4$ & $23.41 \pm 0.12$ & 0.13 & 159 & $0.21 \pm 0.01$ & 1.08 & Y \\
\hline $2322+346$ & $18.20 \pm 0.06$ & $15.15 \pm 0.05$ & $8.4 \pm 0.3$ & $22.99 \pm 0.08$ & 0.25 & 65 & $0.19 \pm 0.01$ & 1.02 & $\mathrm{Y}$ \\
\hline
\end{tabular}




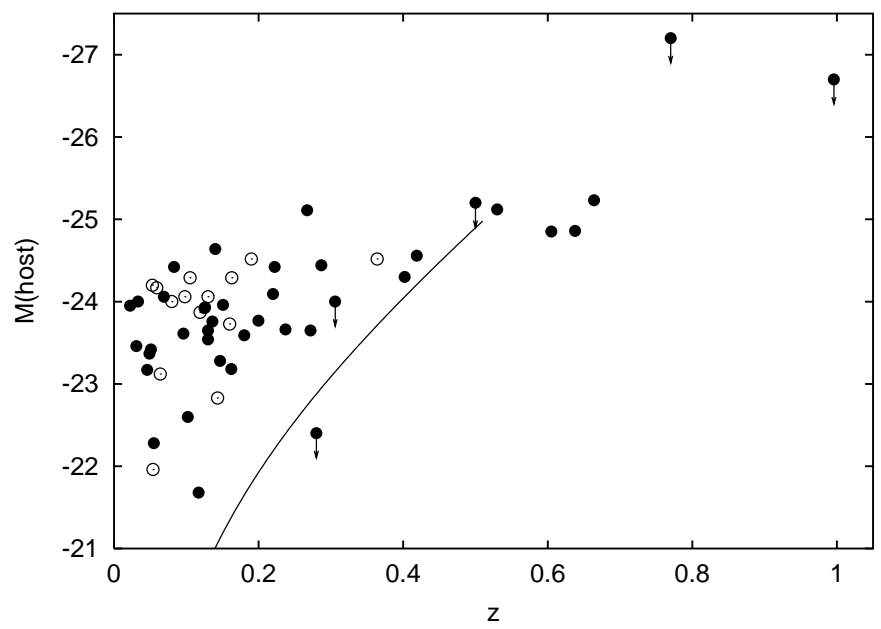

Fig. 4. The dependence of the host galaxy luminosity on redshift. Open symbols refer to BL Lac candidates, filled symbols to confirmed BL Lacs. Arrows mark the objects for which only an upper limit could be determined. The line gives the absolute magnitude of an object with $R=19.0$.

model $(\beta=1.0)$. Only in two cases $(1419+543$ and $1540+147)$ among the 62 resolved objects one obtains a smaller $\chi^{2}$ for the disk model than for the elliptical model. In $1540+147$ the difference is small and the overall fit is not very good $\left(\chi^{2}=16.0\right.$ for the disk model and 16.1 for the elliptical model). Thus we do not believe that the difference is significant.

In $1419+543$ there is evidence of an inner component in the host galaxy (see individual notes on the objects). In addition to the core + bulge fits we also fitted a core + bulge + disk model to this object. The disk component was defined by four parameters, magnitude $\left(m_{\text {disk }}\right)$, effective radius $\left(r_{\text {disk }}\right)$, ellipticity $\left(\epsilon_{\text {disk }}\right)$ and position angle $\left(\mathrm{PA}_{\text {disk }}\right)$. We used $\beta=1.0$ for the disk component and the disk and bulge were assumed to be cocentric. The total number of free parameters was thus 14 (3 for the core, 7 for the bulge and 4 for the disk). In Fig. 6 we show the two models and the corresponding model components. With the core + bulge + disk model we obtain a slightly better fit $\left(\chi^{2}=2.06\right)$ than with the core + bulge model $\left(\chi^{2}=2.31\right)$. The disk is fainter than the bulge $\left(m_{\text {disk }}=17.21\right.$ and $m_{\text {bulge }}=$ $17.02)$, but larger $\left(r_{\text {disk }}=14.7 \mathrm{kpc}\right.$ and $\left.r_{\text {bulge }}=11.6 \mathrm{kpc}\right)$ and its ellipticity is higher $\left(\epsilon_{\text {disk }}=0.50\right.$ and $\left.\epsilon_{\text {bulge }}=0.18\right)$. For the bulge we obtain $\beta=0.16$.

We also performed an isophote analysis using the ellipse task in IRAF. This analysis shows that there is a peak in both ellipticity and in the $b_{4}$ Fourier coefficient (as defined in Jedrzejewski 1987) at $\sim 3$ to 8 arcsec distance from the nucleus (Fig. 6). Positive values of $b_{4}$ indicate disky isophotes thus supporting the presence of a disk component $1419+543$. We also subtracted the disk component from the 2-dim. images and performed the isophote analysis on the subtracted image. Both the ellipticity and the $b_{4}$ coefficient were considerably reduced by the subtraction of the disk component (Fig. 6). There is thus evidence of a significant disk component in $1419+543$. Given the large size of the disk and high disk to bulge luminosity ratio $(D / B)=0.84)$ and the host galaxy of $1419+543$ should
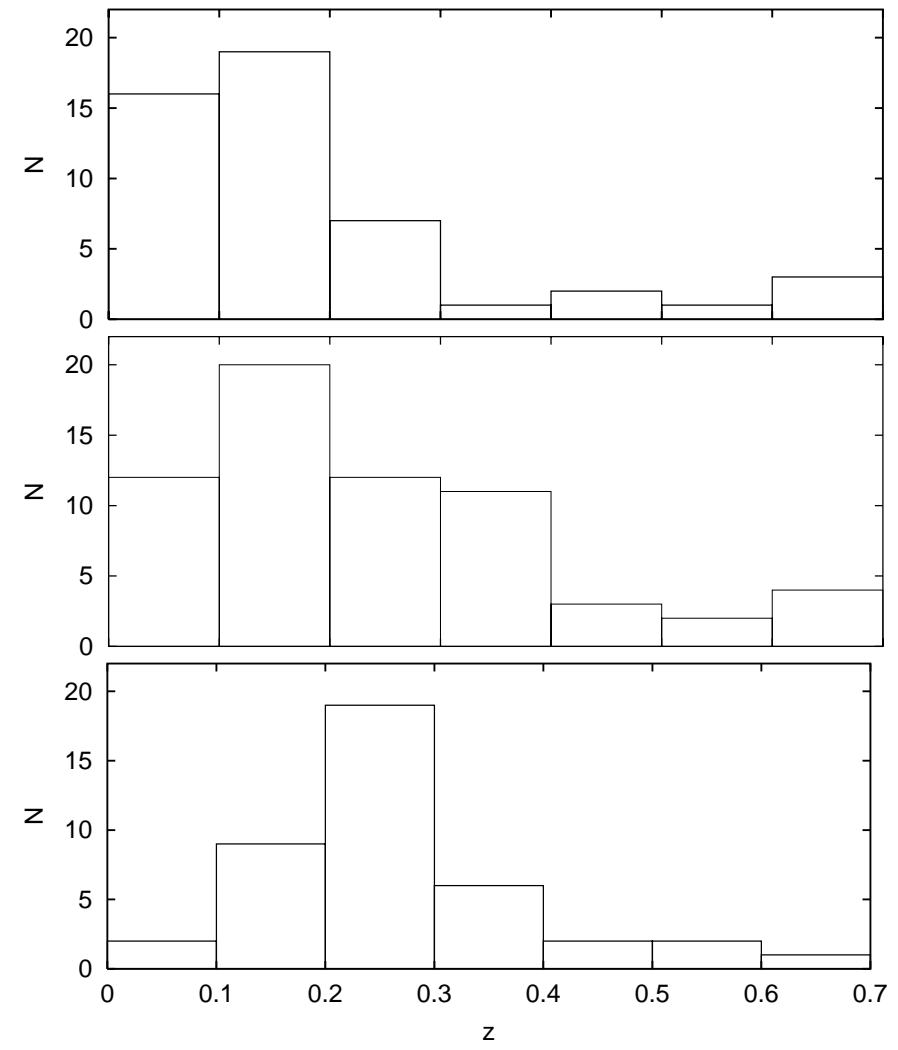

Fig. 5. The redshift distribution of the resolved objects in the RGB sample (upper panel). For comparison the redshift distribution of resolved objects in Urry et al. (2000; middle panel) and Falomo \& Kotilainen (1999; lower panel) are shown.

probably be classified as an S0 galaxy, or maybe even an Sb0 galaxy given the appearance of the residuals in Fig. 11.

We have not determined for each object whether the de Vaucouleurs model is significantly (in a statistical sense) better than the disk model, but the $\chi_{\exp }^{2} / \chi_{\mathrm{deV}}^{2}$. values in Table 4 and the fact that not a single clear case of a disk host was detected strongly support the view that BL Lac objects are almost exclusively found in elliptical galaxies. Urry et al. (1999) found that for 58 objects among the 72 that were resolved the de Vaucouleurs model was preferred over the disk model with a high statistical significance and concluded that at most $8 \%$ of the BL Lacs are hosted by disk type galaxies.

In Fig. 7 we show the distribution of the fitted $\beta$ values for the 55 objects that were judged to be well enough resolved for such a fit. The median $\beta$ is 0.18 , well below $\beta=0.25$ typically used to describe elliptical galaxy profiles. It is well known, that not all ellipticals follow strictly the $\beta=0.25$ intensity profile (e.g. Caon et al. 1993). It is therefore interesting to ask whether the deviation in Fig. 7 is significant. This is currently very difficult to answer given the uncertainties in the determination of $\beta$. There is also a selection effect at work here: only objects that were judged well enough resolved were fit with the free $\beta$ model, which means that bright and/or large host galaxies are favored. Caon et al. (1993) have shown that in normal $\mathrm{E}$ and S0 galaxies brighter and larger galaxies have also higher Sérsic $n$, i.e. smaller $\beta(=1 / n)$. We also see a correlation of this type in our data (Fig. 8), albeit with high scatter. The median 
Table 6. Intrinsic parameters of the objects.

\begin{tabular}{|c|c|c|c|c|c|c|c|c|}
\hline \multirow[b]{2}{*}{ RGB } & \multirow[b]{2}{*}{$K_{R}$} & \multicolumn{3}{|c|}{ 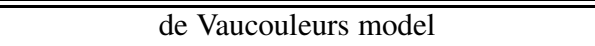 } & \multicolumn{3}{|c|}{ free $\beta$ model } & \multirow[b]{2}{*}{$\begin{array}{l}\text { New } \\
\text { host? }\end{array}$} \\
\hline & & $M_{\text {core }}$ & $M_{\text {host }}$ & $\begin{array}{c}r_{\mathrm{eff}} \\
{[\mathrm{kpc}]}\end{array}$ & $M_{\text {core }}$ & $M_{\text {host }}$ & $\begin{array}{c}r_{\mathrm{eff}} \\
{[\mathrm{kpc}]}\end{array}$ & \\
\hline $0007+472$ & 0.37 & $-22.98 \pm 0.03$ & $>-22.4$ & - & - & - & - & - \\
\hline $0110+418$ & 0.09 & $-20.47 \pm 0.05$ & $-23.61 \pm 0.04$ & $13.6 \pm 0.2$ & $-20.23 \pm 0.05$ & $-23.70 \pm 0.05$ & $15.6 \pm 0.4$ & $\mathrm{Y}$ \\
\hline $0123+343$ & 0.35 & $-23.52 \pm 0.03$ & $-23.65 \pm 0.03$ & $9.1 \pm 0.5$ & $-23.40 \pm 0.04$ & $-23.97 \pm 0.07$ & $10.6 \pm 1.1$ & - \\
\hline $0152+017$ & 0.07 & $-21.78 \pm 0.05$ & $-24.00 \pm 0.05$ & $9.7 \pm 0.2$ & $-21.66 \pm 0.05$ & $-24.09 \pm 0.05$ & $10.9 \pm 0.2$ & $\mathrm{Y}$ \\
\hline $0153+712$ & 0.03 & $-19.03 \pm 0.06$ & $-23.95 \pm 0.05$ & $10.5 \pm 0.2$ & $-17.98 \pm 0.09$ & $-24.25 \pm 0.07$ & $17.6 \pm 1.1$ & $\mathrm{Y}$ \\
\hline $0214+517$ & 0.05 & $-20.81 \pm 0.04$ & $-23.37 \pm 0.04$ & $9.4 \pm 0.1$ & $-20.28 \pm 0.06$ & $-23.83 \pm 0.06$ & $21.3 \pm 1.2$ & Y \\
\hline $0314+247$ & 0.05 & $-19.47 \pm 0.04$ & $-21.96 \pm 0.04$ & $7.2 \pm 0.1$ & $-19.70 \pm 0.04$ & $-21.72 \pm 0.04$ & $5.3 \pm 0.1$ & $\mathrm{Y}$ \\
\hline $0326+024$ & 0.15 & $-23.32 \pm 0.03$ & $-23.28 \pm 0.03$ & $8.3 \pm 0.3$ & $-23.17 \pm 0.04$ & $-23.73 \pm 0.07$ & $12.1 \pm 0.9$ & - \\
\hline $0416+010$ & 0.38 & $-24.61 \pm 0.05$ & $-24.44 \pm 0.05$ & $13.8 \pm 0.5$ & $-24.54 \pm 0.05$ & $-24.78 \pm 0.09$ & $19.8 \pm 1.4$ & - \\
\hline $0654+427$ & 0.12 & $-22.23 \pm 0.04$ & $-23.93 \pm 0.04$ & $13.9 \pm 0.3$ & $-21.87 \pm 0.05$ & $-24.41 \pm 0.06$ & $29.7 \pm 2.0$ & Y \\
\hline $0656+426$ & 0.06 & $-20.85 \pm 0.05$ & $-24.17 \pm 0.05$ & $14.5 \pm 0.1$ & $-19.53 \pm 0.08$ & $-24.60 \pm 0.05$ & $30.8 \pm 0.9$ & $\mathrm{Y}$ \\
\hline $0710+591$ & 0.12 & $-21.91 \pm 0.03$ & $-23.92 \pm 0.03$ & $13.6 \pm 0.3$ & $-21.52 \pm 0.05$ & $-24.27 \pm 0.04$ & $22.3 \pm 1.1$ & - \\
\hline $0831+044$ & 0.20 & $-24.82 \pm 0.03$ & $-23.59 \pm 0.04$ & $10.3 \pm 1.0$ & $-24.76 \pm 0.04$ & $-24.27 \pm 0.09$ & $21.8 \pm 3.4$ & - \\
\hline $0854+201$ & 0.42 & $-26.38 \pm 0.05$ & $>-24.0$ & - & - & - & - & - \\
\hline $0916+526$ & 0.21 & $-22.10 \pm 0.05$ & $-24.52 \pm 0.05$ & $19.6 \pm 0.4$ & $-21.89 \pm 0.06$ & $-24.70 \pm 0.05$ & $25.6 \pm 0.8$ & Y \\
\hline $0928+747$ & 1.38 & $-23.35 \pm 0.07$ & $-24.86 \pm 0.06$ & $10.1 \pm 1.6$ & $-23.30 \pm 0.09$ & $-24.99 \pm 0.10$ & $10.8 \pm 1.5$ & - \\
\hline $1000+225$ & 0.70 & $-23.75 \pm 0.05$ & $-24.56 \pm 0.05$ & $22.1 \pm 1.3$ & $-23.67 \pm 0.05$ & $-24.94 \pm 0.09$ & $38.9 \pm 3.4$ & $\mathrm{Y}$ \\
\hline $1012+424$ & 0.55 & $-24.20 \pm 0.03$ & $-24.52 \pm 0.04$ & $24.9 \pm 1.3$ & $-24.14 \pm 0.03$ & $-25.01 \pm 0.09$ & $56.3 \pm 7.2$ & $\mathrm{Y}$ \\
\hline $1015+494$ & 0.23 & $-24.40 \pm 0.03$ & $-23.77 \pm 0.03$ & $9.1 \pm 0.4$ & $-24.26 \pm 0.04$ & $-24.46 \pm 0.08$ & $15.1 \pm 1.9$ & - \\
\hline $1053+494$ & 0.14 & $-22.04 \pm 0.07$ & $-24.64 \pm 0$ & & & & & Y \\
\hline $1104+382$ & 0.03 & $-23.51 \pm 0.05$ & $-23.46 \pm 0.05$ & $8.4 \pm 0.2$ & $-23.53 \pm 0.05$ & $-23.27 \pm 0.05$ & $6.3 \pm 0.1$ & - \\
\hline $1136+676$ & 0.14 & $-21.81 \pm 0.10$ & $-23.76 \pm 0.10$ & $10.1 \pm 0.3$ & $-21.51 \pm 0.11$ & $-23.97 \pm 0.10$ & $12.5 \pm 0.3$ & Y \\
\hline $1136+701$ & 0.05 & $-21.50 \pm 0.02$ & $-23.17 \pm 0.02$ & $8.5 \pm 0.1$ & $-21.39 \pm 0.03$ & $-23.30 \pm 0.03$ & $10.2 \pm 0.4$ & - \\
\hline $1149+246$ & 0.65 & $-23.11 \pm 0.04$ & $-24.30 \pm 0.03$ & $7.3 \pm 0.7$ & $-22.87 \pm 0.05$ & $-24.53 \pm 0.05$ & $7.4 \pm 0.7$ & Y \\
\hline & 0.13 & $-24.82 \pm 0.05$ & $-23.54 \pm 0.05$ & & & $-24.44 \pm 0.31$ & $57.7 \pm$ & - \\
\hline $1221+282$ & 0.10 & $-24.84 \pm 0.10$ & $-22.60 \pm 0.10$ & $4.8 \pm 0.9$ & $-24.81 \pm 0.10$ & $-23.18 \pm 0.22$ & $7.8 \pm 0.9$ & - \\
\hline $1310+323$ & 2.51 & $-26.88 \pm 0.02$ & $>-26.7$ & - & - & - & - & - \\
\hline $1341+399$ & 0.17 & $-21.78 \pm 0.07$ & $-24.29 \pm 0.07$ & $21.2 \pm 0.6$ & $-21.67 \pm 0.07$ & $-24.43 \pm 0.08$ & $26.2 \pm 1.2$ & $\mathrm{Y}$ \\
\hline $1419+543$ & 0.15 & $-24.47 \pm 0.02$ & $-23.96 \pm 0.02$ & $12.6 \pm 0.6$ & $-24.51 \pm 0.02$ & $-23.63 \pm 0.02$ & $10.4 \pm 0.3$ & - \\
\hline $1427+541$ & 0.10 & $-19.93 \pm 0.02$ & $-24.29 \pm 0.02$ & $21.6 \pm 0.2$ & $-20.23 \pm 0.02$ & $-24.13 \pm 0.03$ & $16.8 \pm 0.5$ & $\mathrm{Y}$ \\
\hline $1428+426$ & 0.13 & $-22.29 \pm 0.02$ & $-23.65 \pm 0.02$ & $9.3 \pm 0.3$ & $-22.26 \pm 0.02$ & $-23.69 \pm 0.03$ & $9.5 \pm 0.3$ & - \\
\hline $1442+120$ & 0.17 & $-22.63 \pm 0.02$ & $-23.18 \pm 0.02$ & $7.9 \pm 0.3$ & $-22.60 \pm 0.02$ & $-23.27 \pm 0.03$ & $8.9 \pm 0.3$ & - \\
\hline $1516+293$ & 0.13 & $-20.72 \pm 0.02$ & $-24.06 \pm 0.02$ & $14.0 \pm 0.3$ & $-20.56 \pm 0.04$ & -24.13 & $15.5=$ & $\mathrm{Y}$ \\
\hline $1532+302$ & 0.06 & $-19.85 \pm 0.02$ & $-23.12 \pm 0.02$ & $8.5 \pm 0.2$ & $-19.20 \pm 0.04$ & $-23.38 \pm 0.02$ & $13.0 \pm 0.5$ & $\mathrm{Y}$ \\
\hline $1534+372$ & 0.14 & $-21.66 \pm 0.02$ & $-22.83 \pm 0.02$ & $6.5 \pm 0.3$ & $-21.54 \pm 0.02$ & $-22.97 \pm 0.03$ & $7.2 \pm 0.3$ & $\mathrm{Y}$ \\
\hline $1540+147$ & 1.27 & $-26.06 \pm 0.02$ & $-24.85 \pm 0.05$ & $26.7 \pm 4.4$ & - & - & - & - \\
\hline $1554+201$ & 0.26 & $-22.95 \pm 0.06$ & $-24.42 \pm 0.05$ & $12.4 \pm 0.4$ & $-22.95 \pm 0.08$ & $-24.41 \pm 0.08$ & $12.4 \pm 0.4$ & - \\
\hline $1626+352$ & 0.93 & $-24.30 \pm 0.04$ & $>-25.2$ & - & - & - & - & - \\
\hline $1644+457$ & 0.26 & $-22.15 \pm 0.06$ & $-24.09 \pm 0.06$ & $12.5 \pm 0.9$ & $-22.13 \pm 0.08$ & $-24.12 \pm 0.08$ & $13.0 \pm 0.9$ & Y \\
\hline $1653+397$ & 0.04 & $-22.41 \pm 0.04$ & $-24.00 \pm 0.04$ & $10.4 \pm 0.2$ & $-22.12 \pm 0.04$ & $-24.66 \pm 0.06$ & $35.8 \pm 3.3$ & - \\
\hline $1728+502$ & 0.05 & $-21.17 \pm 0.03$ & $-22.28 \pm 0.02$ & $5.2 \pm 0.1$ & $-21.11 \pm 0.04$ & $-22.32 \pm 0.04$ & $5.3 \pm 0.1$ & - \\
\hline $1743+195$ & 0.08 & $-22.46 \pm 0.02$ & $-24.42 \pm 0.03$ & $20.0 \pm 0.5$ & $-22.32 \pm 0.02$ & $-24.82 \pm 0.06$ & $40.4 \pm 3.8$ & - \\
\hline $1745+398$ & 0.34 & $-23.02 \pm 0.03$ & $-25.11 \pm 0.04$ & $29.4 \pm 0.5$ & $-22.84 \pm 0.03$ & $-25.61 \pm 0.06$ & $70.1 \pm 4.9$ & $\mathrm{Y}$ \\
\hline $1748+700$ & 1.83 & $-28.04 \pm 0.05$ & $>-27.2$ & - & - & - & - & - \\
\hline $1750+470$ & 0.17 & $-20.87 \pm 0.04$ & $-23.73 \pm 0.04$ & $14.7 \pm 0.3$ & $-20.72 \pm 0.04$ & $-23.82 \pm 0.05$ & $17.0 \pm 1.0$ & Y \\
\hline $1806+698$ & 0.05 & $-22.70 \pm 0.03$ & $-23.42 \pm 0.03$ & $11.3 \pm 0.1$ & $-22.59 \pm 0.03$ & $-23.96 \pm 0.05$ & $29.0 \pm 2.1$ & - \\
\hline $1813+317$ & 0.11 & $-22.59 \pm 0.05$ & $-21.68 \pm 0.05$ & $7.1 \pm 0.7$ & $-22.56 \pm 0.05$ & $-21.97 \pm 0.11$ & $9.7 \pm 1.2$ & $\mathrm{Y}$ \\
\hline $1824+568$ & 1.47 & $-25.71 \pm 0.04$ & $-25.23 \pm 0.06$ & $23.2 \pm 1.9$ & - & - & - & - \\
\hline $1841+591$ & 1.02 & $-23.51 \pm 0.02$ & $-25.12 \pm 0.03$ & $15.4 \pm 0.7$ & $-23.53 \pm 0.03$ & $-25.05 \pm 0.10$ & $14.7 \pm 1.5$ & $\mathrm{Y}$ \\
\hline $2039+523$ & 0.05 & $-20.71 \pm 0.03$ & $-24.20 \pm 0.03$ & $11.6 \pm 0.3$ & $-20.46 \pm 0.03$ & $-24.41 \pm 0.04$ & $15.9 \pm 0.9$ & - \\
\hline $2145+073$ & 0.29 & $-23.03 \pm 0.06$ & $-23.66 \pm 0.06$ & $11.0 \pm 0.8$ & $-23.02 \pm 0.07$ & $-23.67 \pm 0.10$ & $11.0 \pm 0.8$ & - \\
\hline $2202+422$ & 0.06 & $-24.11 \pm 0.07$ & $-24.06 \pm 0.07$ & $11.2 \pm 0.4$ & $-24.09 \pm 0.07$ & $-24.22 \pm 0.09$ & $14.3 \pm 1.1$ & - \\
\hline $2250+384$ & 0.12 & $-22.65 \pm 0.02$ & $-23.87 \pm 0.03$ & $15.6 \pm 0.5$ & $-22.62 \pm 0.02$ & $-23.99 \pm 0.05$ & $18.6 \pm 1.1$ & $\mathrm{Y}$ \\
\hline $2322+346$ & 0.09 & $-21.28 \pm 0.05$ & $-24.06 \pm 0.05$ & $14.4 \pm 0.2$ & $-21.07 \pm 0.06$ & $-24.21 \pm 0.05$ & $18.0 \pm 0.6$ & $\mathrm{Y}$ \\
\hline
\end{tabular}



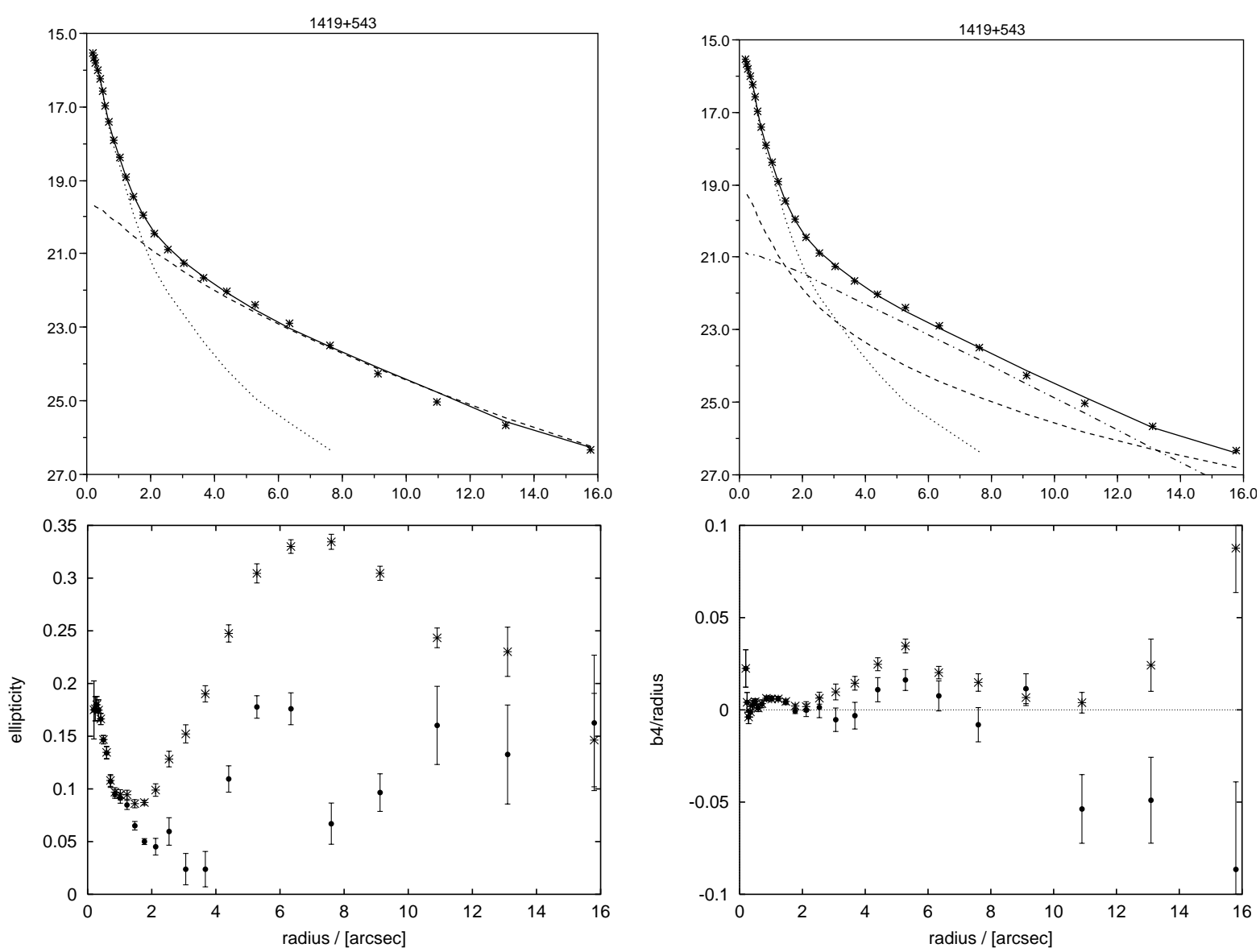

Fig. 6. Surface brightness analysis of $1419+543$. The upper panels show the observed surface brightness (asterisks) and two models (solid lines): the core+ bulge model (left) and the core + bulge + disk model (right). The core model (dotted line), bulge model (dashed line) and disk model(dot-dashed line) are also shown. The lower panels show the ellipticity and $b_{4}$ Fourier coefficient. Asterisks mark observer values and filled symbols the values after subtraction of the disk component.

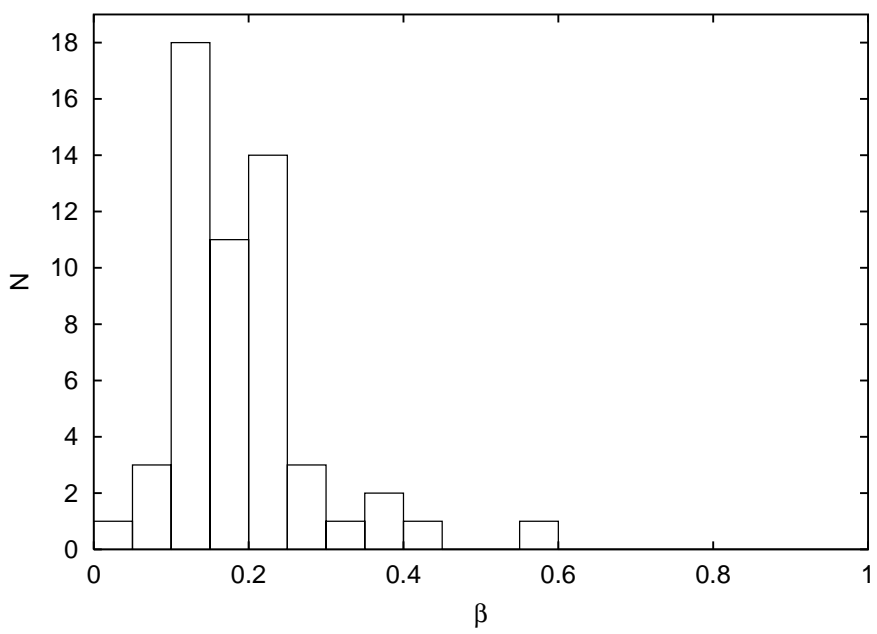

Fig. 7. The distribution of $\beta$ values.

size of the free $\beta$ fitted galaxies is $15.5 \mathrm{kpc}$ which, applying the relationship $\log n=0.28+0.52 \log r_{\mathrm{e}}$ in Caon et al. (1993), would translate to $\beta=0.13$, i.e. significantly lower than 0.25 .

There could also be factors related to the fitting process that affect the $\beta$ distribution. First, since the free $\beta$ model has more freedom, it is harder to separate the host galaxy

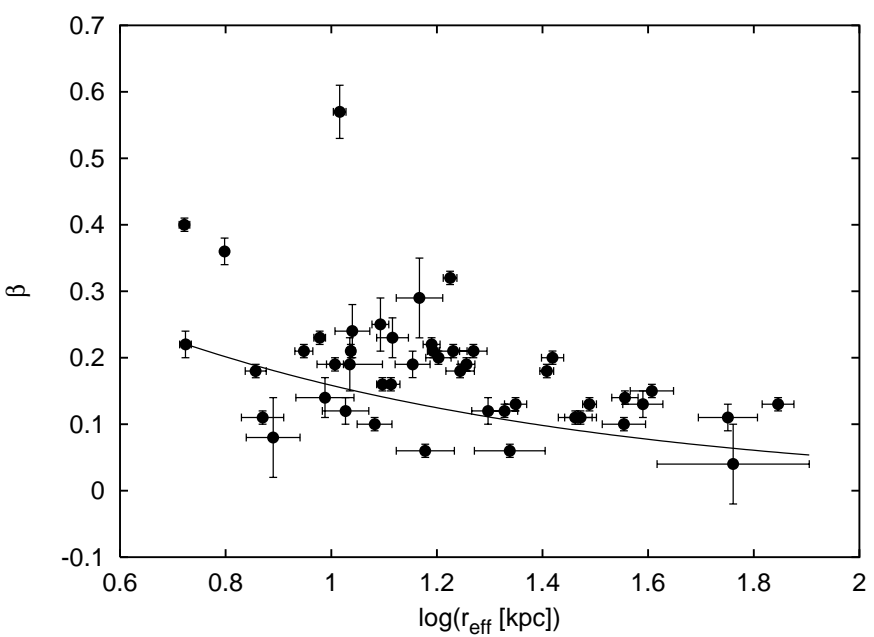

Fig. 8. The dependence of $\beta$ on effective radius. The solid line gives the relationship in Caon et al. (1993) for normal E and S0 galaxies.

contribution from the nuclear contribution than with the more rigid de Vaucouleurs model, especially in the areas close to the nucleus. We have compared the fitted $\beta$ in our error simulations (Sect. 5) to the input values and found no strong bias with respect to $\beta$. The median difference $\beta_{\text {input }}-\beta_{\text {output }}$ is -0.01 with a 


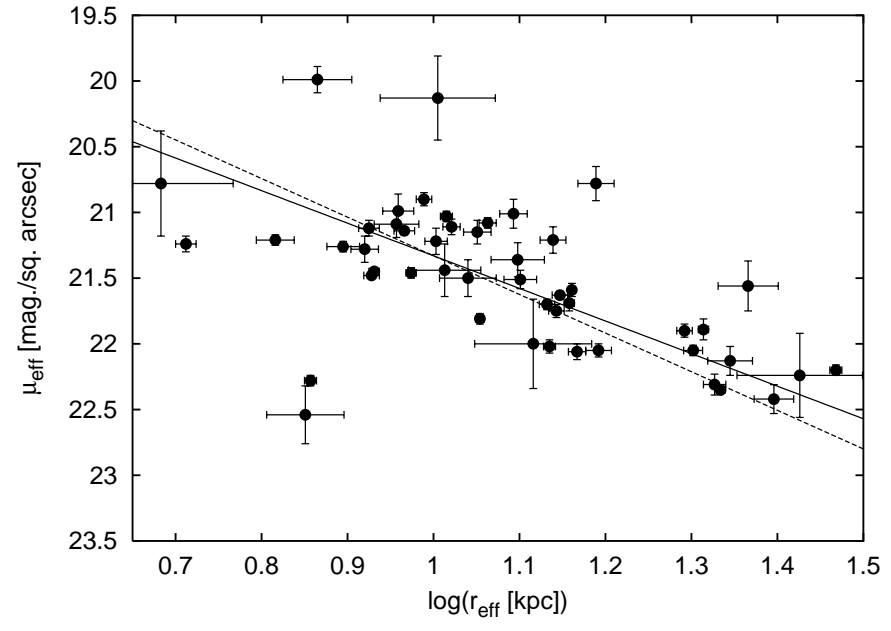

Fig. 9. The Kormendy relation for the host galaxies when the de Vaucouleurs model is used. The solid line gives the fitted slope for the data, the dashed line gives the relationship for normal ellipticals from Hamabe \& Kormendy (1987).

$0.02 \mathrm{rms}$ deviation i.e. if there is any bias, it is actually making the fitted $\beta$ larger than the "correct" $\beta$.

Secondly, there is the possibility that the companion galaxies that are commonly seen around these BL Lacs could somehow decrease $\beta$. Lower $\beta$ means that there is more light in the outer parts of the galaxy. This light could partially be light from nearby companions that we cannot mask out completely. To study the effect of the companions we made an examination of the BL Lac images and divided them into two groups: those that seemed to possess at least one relatively bright companion inside the fitting radius and those that did not. Of the $55 \mathrm{ob}-$ jects fitted with the free $\beta$ model 23 were judged to belong to the first group and 32 to the second. We found the median $\beta$ to be 0.18 in both groups, i.e. the same as for the whole sample. This simple test thus suggests, that we are able to mask the companions sufficiently to not seriously affect the fitted $\beta$. A better solution than just mask the companions might be to fit and remove them iteratively. Recently McLure et al. (1999) fitted free $\beta$ models to a sample of radio galaxies, radio-quiet quasars and radio-loud quasars observed with the HST. They found the $\beta$ values to be concentrated around $\beta=0.25$. The luminosities and sizes of the host galaxies studied by McLure et al. (1999) are similar to ours, so the difference in the average values of $\beta$ remains unexplained.

Many objects show considerable residuals in the inner parts of the host galaxy that can not be attributed to PSF errors or complications arising from nearby bright companions alone (e.g. $0152+017,0656+426,1419+543)$. In these objects there is probably an inner component, possibly a disk, and a two-component model for the host galaxy would be better. However, a three-component model (core + bulge + disk $)$ would complicate the task even further and, given the small apparent size of many of the objects, it is not clear if the model parameters could be sufficiently constrained.

In Fig. 9 we show the $\mu_{\text {eff }}-r_{\text {eff }}$ projection of the fundamental plane (the Kormendy relation) for the resolved BL Lacs with known redshifts when the de Vaucouleurs model is used for the host galaxy. The values of $\mu_{\text {eff }}$ in Tables 4 and 5 have been corrected for the galactic extinction, K-correction and the cosmological surface brightness dimming. For the de Vaucouleurs model (Fig. 9) the best-fitting line is given by $\mu_{\text {eff }}=(18.85 \pm$ $0.33)+(2.48 \pm 0.27) \log \left(r_{\text {eff }}\right)$ and for the free $\beta$ model $\mu_{\text {eff }}=$ $(17.50 \pm 0.49)+(3.91 \pm 0.38) \log \left(r_{\text {eff }}\right)$. The former relationship is similar to the relationship in Hamabe \& Kormendy (1987), who fitted the de Vaucouleurs model to a sample of nonactive elliptical and early-type disk galaxies. The slope for the free $\beta$ model is somewhat steeper, but within the range found for other BL Lacs samples. FK99 found $\mu_{\mathrm{eff}}=16.45+4.6 \log \left(r_{\mathrm{eff}}\right)$, Urry et al. $(2000) \mu_{\text {eff }}=(17.2 \pm 0.7)+(3.9 \pm 0.9) \log \left(r_{\text {eff }}\right)$ and $\mathrm{P} 02 \mu_{\text {eff }}=(17.85 \pm 0.68)+(3.27 \pm 0.59) \log \left(r_{\text {eff }}\right)$. Moreover, the combined sample of FR I and II radio galaxies studied by Govoni et al. (2000) gave $\mu_{\text {eff }}=(18.44 \pm 0.35)+(2.58 \pm 0.29)$ $\log \left(r_{\text {eff }}\right)$. All these relationships are very similar and, like many previous authors, we can conclude that in the $\mu_{\text {eff }}-r_{\text {eff }}$ plane the BL Lac host galaxies are indistinguishable from normal ellipticals and radio galaxies.

There are a few objects above or below the general trend in Fig. 9. The two objects below the main part at $\log \left(r_{\text {eff }}\right) \sim 0.85$ are $1813+317$ (the object with larger error bars) and $0314+247$. Both objects have been discussed in Sect. 5.2. The redshift of $1813+317$ could be actually higher than what is quoted in the literature and $0314+247$ is clearly affected by dust which probably lowers its $\mu_{\text {eff }}$. The four objects clearly above the main part in Fig. 9 are all high-redshift objects: these are 1149+246 $(z=0.402), 0928+747(z=0.638), 1841+591(z=0.530)$ and $1824+568(z=0.664)$ from upper left to lower right. All are intrinsically very bright and may thus not be representative of the whole elliptical galaxy class. Their large K-corrections (0.6-1.5) lift them above other objects and if their SEDs are not similar to elliptical galaxy SEDs, as we have assumed, our correction would be incorrect and they could actually lie lower in Fig. 9. We also do not correct for passive evolution of the host galaxies, which, if applied, would bring them lower in Fig. 9.

There are a handful of objects that are promising lensing candidates. The two double sources $(0035+598$ and $2319+161)$ are probably not lensed: there is no sign of the lensing galaxy in either object. In $0035+598$ the colors of the two point objects are very different (FK99) and our spectrum shows the nearby object of $2319+161$ to be a galactic star. In $0814+296$ there are two very nearby objects and in $0915+295$ our resolution is not sufficient to determine whether there is a nearby companion or a decentered host. In $0250+172$ there is a faint object very close to the nucleus but the nature of this objects is unclear.

Finally, as was noted in Sect. 2, only $50 \%$ of the RGB BL Lacs have a spectroscopically determined redshift, and in some cases the redshift is uncertain. During this study we found altogether 14 resolved objects that do not currently have a redshift. We have started a program to determine the redshifts of these objects and for one object $(2039+523, z=0.053)$ a redshift has already been obtained. In Table 2 we show the estimated redshifts for the remaining 13 objects. We used the fitted host magnitude, effective radius and ellipticity to plot the object's location in the $\log \left(r_{\text {eff }}\right)-\mu_{\text {eff }}$ plane as a function of redshift. At some point this curve intersects with the fitted Kormendy relation in Fig. 9 and in Table 2 we give the redshift corresponding 
$1151+589$

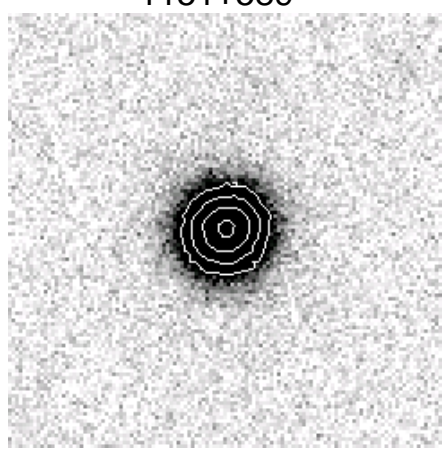

22.9" 21.5

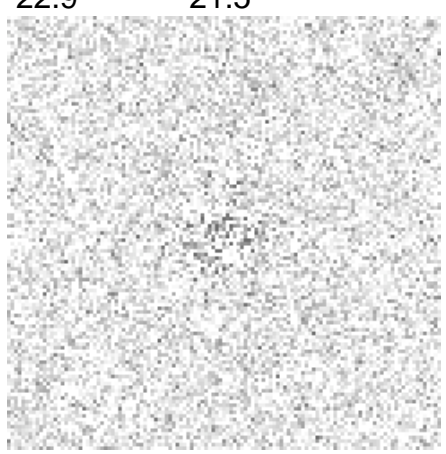

$0109+182$

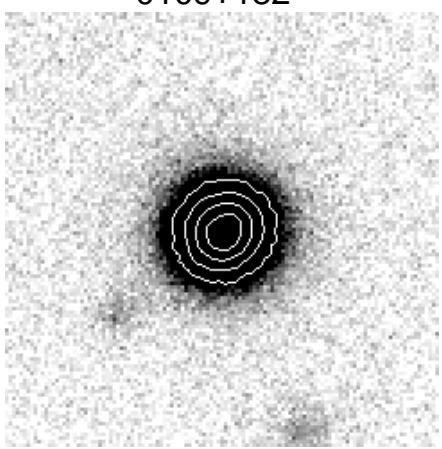
22.0

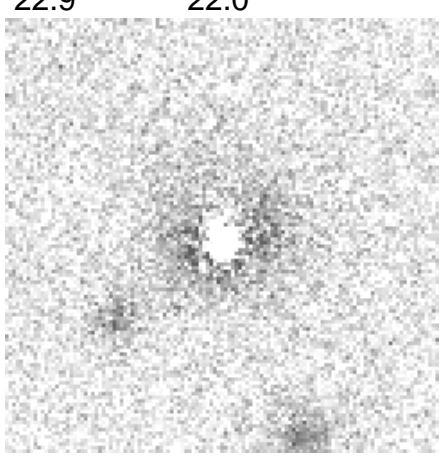

$0929+502$

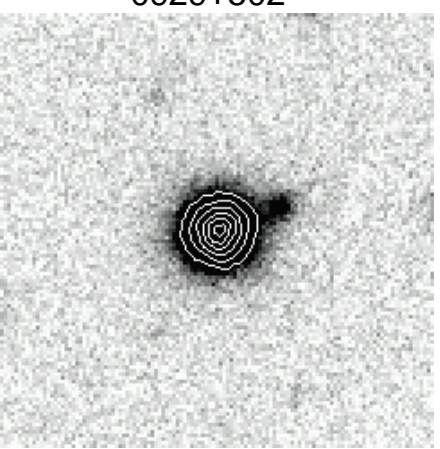

22.5

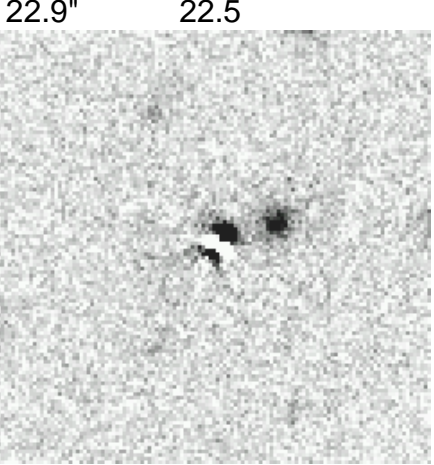

$2322+346$
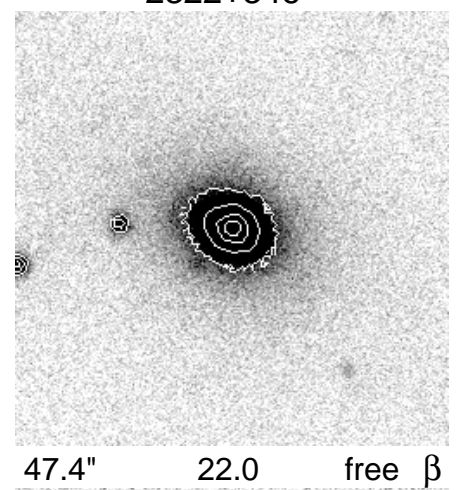

Fig. 10. Examples of objects in the RGB BL Lac sample. The upper panel for each object shows the observed image and the lower panel the model subtracted image. North is up and east is to the left in all images. Between the panels (from left to right) the field of view in arcsec, surface brightness of the lowest contour (mag/sq arcsec) and the galaxy model used in the subtraction are given. Contour interval is $1 \mathrm{mag} / \mathrm{sq}$ arcsec. If no galaxy model is given, only a point source has been subtracted from the observed image. The upper two objects are unresolved leaving either no residuals or PSF residuals due to the different focus of the object and the PSF star (see the text) after PSF subtraction. The lower left panel shows an object with faint emission around the nucleus, but this object is still unresolved according to the criteria used in this paper. The lower right panel shows a clearly resolved object. 

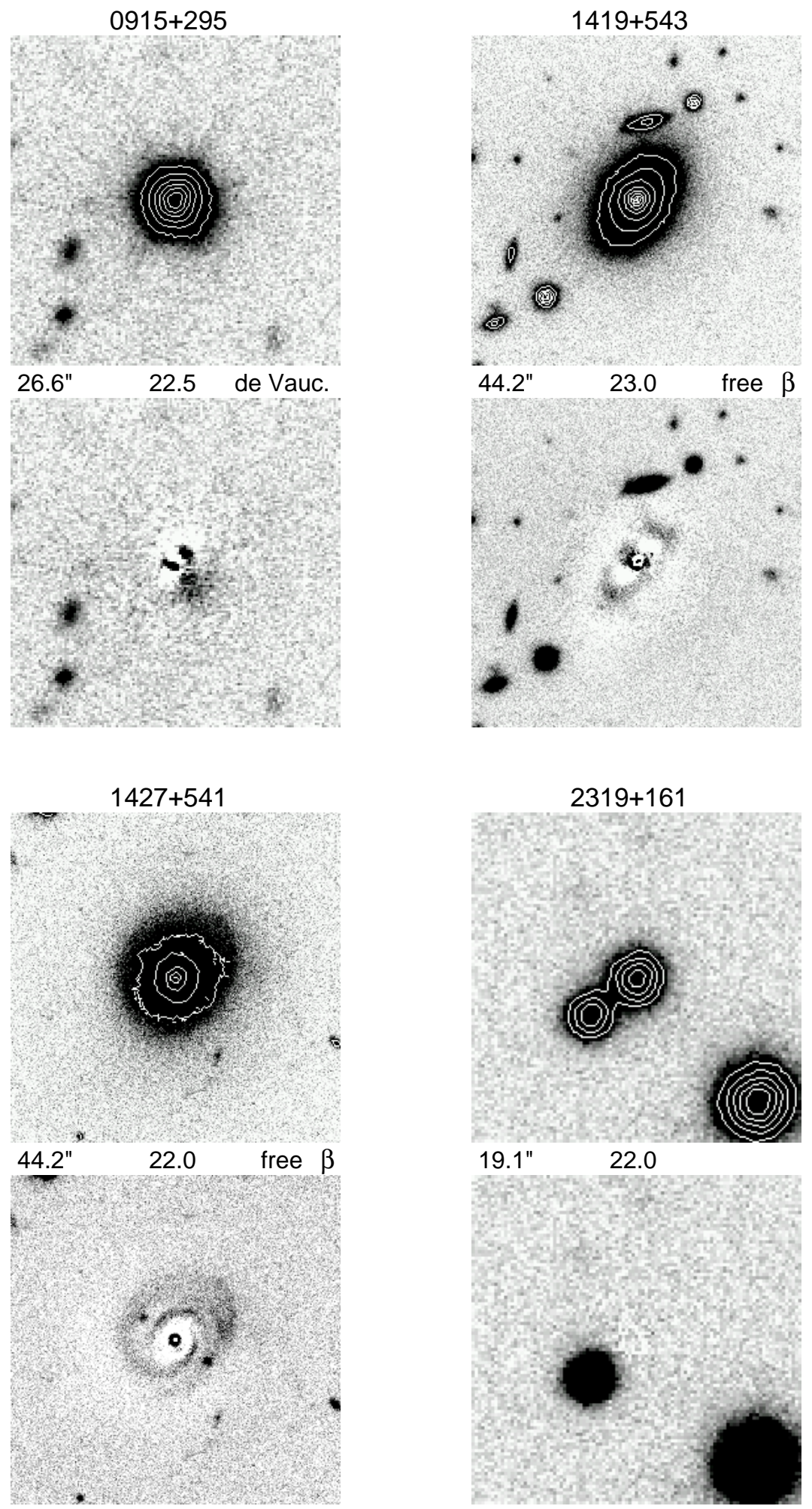

Fig. 11. Examples of unusual objects : in $0915+295$ a very nearby companion or an intervening galaxy is seen, $1419+253$ has unusual residuals, 1427+541 has spiral arms and 2319+161 has a nearby companion point source, shown to be an M-star by spectroscopy.

to this intersect point for each object. We tested this method with 13 objects with known redshifts between $0.1-0.6$ and found the estimated redshifts to be accurate to within $\sim 0.05$, except for two high- $z$ objects that lie above the main part in Fig. 9. Thus some objects in Table 2 may have redshifts higher than indicated if their host galaxies are somehow unusual. Finally, we note that the objects in Table 2 have relatively high redshifts and if they are added to our sample, the redshift distibution of the resolved objects becomes more similar to that of Urry et al. (2000) and Falomo \& Kotilainen (1999). 


\section{Summary}

We have obtained new $R$-band images of 86 BL Lacertae objects in the ROSAT-Green Bank (RGB) sample. Combining the data from our previous studies we present here the optical images and host galaxy analysis of 100 objects. For 62 objects in the sample we did not find previously published data in the literature, 35 of these were new BL Lacs discovered during the RGB BL Lac survey. We were able to resolve the host galaxy in $62 \%$ of the objects. We analyze the host galaxies using a twodimensional fitting procedure and give the best-fit core magnitude and host galaxy parameters (magnitude, effective radius, ellipticity, position angle and $\beta$ ) for each object.

With two exceptions, all objects are formally better fit by a model representing an elliptical galaxy $(\beta=0.25)$ than by a disk galaxy model $(\beta=1.0)$. However, the disk host fit is only marginally better in the two exceptions. One of them $(1419+543)$ has a very large disk component, and should probably be classified as an S0 or Sb0 galaxy. Thus we do not find a single clear case of a disk-dominated host galaxy in our sample.

The host galaxies have an average brightness $M_{R}=-23.9 \pm$ 0.8 and effective radius $r_{\mathrm{eff}}=13.2 \pm 0.8 \mathrm{kpc}$ and their bulk properties are indistinguishable from normal ellipticals and radio galaxies. The median $\beta$ is 0.18 , well below the value of 0.25 that is normally used to describe ellipticals. However, the difference may be due to a selection effect caused by the $M_{R}-\beta$ correlation (Caon et al. 1993).

In a subsequent paper (Nilsson et al., in preparation) we will present a more detailed statistical study of the relationship between BL Lac host galaxies and other ellipticals and the host galaxy - BL Lac nucleus connection.

Acknowledgements. This work has been supported by the Academy of Finland through project 42697 (KN) and through SFB 328 and 439 (JH). KN thanks Prof. I. Appenzeller, Prof. H. Jørgesen, Prof. N. H. Andersen and the staff of Landessternwarte Heidelberg, Copenhagen University Observatory and Risø National Laboratory for support and pleasant working environment during this work. We also thank R. Rekola and P. Keinänen for the help during the observations. The data presented here have been partly taken using ALFOSC, which is owned by the Instituto de Astrofisica de Andalucia (IAA) and operated at the Nordic Optical Telescope under agreement between IAA and the NBIfAFG of the Astronomical Observatory of Copenhagen.

\section{References}

Abraham, R. G., McHardy, I. M., \& Crawford, C. S. 1991, MNRAS, 252,482

Bauer, F. E., Condon, J. J., Thuan, T. X., \& Broderick, J. J. 2000, ApJS, 129, 547

Beckmann, V., Bade, N., \& Wucknitz, O. 1999, A\&A, 352, 395

Benítez, E., Dultzin-Hacyan, D., Heidt, J., et al. 1996, ApJ, 464, L47 Brinkmann, W., Siebert, J., Reich, W., et al. 1995, A\&AS, 109, 147

Caon, N., Capaccioli, M., \& D’Onofrio, M. 1993, MNRAS, 265, 1013 Falomo, R. 1996, MNRAS, 283, 241

Falomo, R., \& Kotilainen, J. K. 1999, A\&A, 352, 85 (FK99)

Falomo, R., Kotilainen, J. K., \& Treves, A. 2002, ApJ, 569, L35

Falomo, R., Melnick, J., \& Tanzi, E. G. 1990, Nature, 345, 692

Falomo, R., Scarpa, R., Treves, A., \& Urry, C. M. 2000, ApJ, 542, 731

Falomo, T., \& Tanzi, E. G. 1991, AJ, 102, 1294

Falomo, R., Urry, C. M., Pesce, J. E., et al. 1997, ApJ, 476, 113
Fanaroff, B., \& Riley, J. M. 1974, MNRAS, 167, 31

Fiorucci, M., \& Tosti, G. 1996, A\&AS, 116, 403

Fiorucci, M., Tosti, G., \& Rizzi, N. 1998, PASP, 110, 105

Ferrarese, L., \& Merritt, D. 2000, ApJ, 539, L9

Fukugita, M., Shimasaku, \& K., Ichikawa, T. 1995, PASP, 107, 945

Gebhardt, K., Bender, R., Bower, G., et al. 2000, ApJ, 539, L13

Giommi, P., Tagliaferri, G., Beuermann, K., et al. 1991, ApJ, 378, 77

Gladders, M. D., Abraham, R. G., McHardy, I. M., et al. 1997, MNRAS, 284, 27

González-Serrano, J. I., \& Carballo, R. 2000, A\&AS, 142, 353

Gorham, P. W., van Zee, L., Unwin, S. C., \& Jacobs, C. 2000, AJ, 119, 1677

Govoni, F., Falomo, R., Fasano, G., \& Scarpa, R. 2000, A\&A, 353, 507

Graham, A. W., Erwin, P., Caon, N., \& Trujillo, I. 2001, ApJ, 563, L11

Gregory, P. C., Scott, W. K., Douglas, K., \& Condon, J. J. 1996, ApJS, 103,427

Hamabe, M., \& Kormendy, J. 1987, in Structure and Dynamics of Elliptical Galaxies, ed. P. T. de Zeeuw (Dordrecht: Kluwer), IAU Symp., 127, 379

Heidt, J., Nilsson, K., Sillanpää, A., Takalo, L. O., \& Pursimo, T. 1999a, A\&A, 341, 683

Heidt, J., Nilsson, K., Fried, J. W., Takalo, L. O., \& Sillanpää, A. 1999b, A\&A, 348, 113

Heidt, J., Nilsson, K., Appenzeller, I., et al. 1999c, A\&A, 352, L11

Iglesias, I. 1998, Appl. Opt., 37, 5427

Jannuzi, B. T., Yanny, B., \& Impey, C. 1997, ApJ, 491, 146

Jansen, R. A., Franx, M., Fabricant, D., \& Caldwell, N. 2000, ApJS, 126, 271

Jedrzejewski, R. I. 1987, MNRAS, 226, 747

Kock, A., Meisenheimer, K., Brinkmann, W., Neumann, M., \& Siebert, J. 1996, A\&A, 307, 745

Kotilainen, J. K., Falomo, R., \& Scarpa, R. 1998, A\&A, 336, 479

Kotilainen, J. K., \& Ward, M. J. 1994, MNRAS, 266, 953

Kotilainen, J. K., Ward, M. J., \& Williger, G. M. 1993, MNRAS, 263, 655

Landolt, A. U. 1992, AJ, 104, 340

Laurent-Muehleisen, S. A., Kollgaard, R. I., Ciardullo, R., et al. 1998, ApJS, 118, 127 (LM98)

Laurent-Muehleisen, S. A., Kollgaard, R. I., Feigelson, E. D., Brinkmann, W., \& Siebert, J. 1999, ApJ, 525, 127 (LM99)

Magorrian, J., Tremaine, S., Richstone, D., et al. 1998, AJ, 115, 2285

Marchã, M. J. M., Browne, I. W. A., Impey, C. D., \& Smith, P. S. 1996, MNRAS, 281, 425

Martel, A. R., Baum, S. A., Sparks, W. B., et al. 1999, ApJS, 122, 81

McHardy, I. M., Luppino, G. A., George, I. M., Abraham, R. G., \& Cooke, B. A. 1992, MNRAS, 256, 655

McLure, R. J., \& Dunlop, J. S. 2001, MNRAS, 327, 199

McLure, R. J., \& Dunlop, J. S. 2002, MNRAS, 331, 795

McLure, R. J., Kukula, M. J., Dunlop, J. S., et al. 1999, MNRAS, 308, 377

Merritt, D., \& Ferrarese, L. 2001, MNRAS, 320, L30

Nilsson, K., Heidt, J., Pursimo, T., Sillanpää, A., \& Takalo, L. O. 1997, ApJ, 484, L107

Nilsson, K., Pursimo, T., Takalo, L. O., et al. 1999a, PASP, 111, 1223

Nilsson, K., Sillanpää, A., \& Takalo, L. O. 1995, in Workshop of two years of intensive monitoring of OJ 287 and 3C 66A, ed. L. O. Takalo, Tuorla Observatory Reports, No. 176, 80

Nilsson, K., Takalo, L. O., Pursimo, T., et al. 1999b, A\&A, 343, 81

Pesce, J. E., Falomo, R., \& Treves, A. 1995, AJ, 110, 1554

Pursimo, T., Nilsson, K., Takalo, L. O., et al. 2002, A\&A, 381, 810 (P02)

Rasmussen, P. K. 1996, ALFOSC installation report, p. 9, obtainable from http://not.iac.es 
Romanishin, W. 1992, ApJ, 401, 65

Scarpa, R., Urry, C. M., Falomo, R., Pesce, J. E., \& Treves, A. 2000a, ApJ, 532, 740 (SU00)

Scarpa, R., Urry, C. M., Falomo, R., et al. 1999a, ApJ, 521, 134

Scarpa, R., Urry, C. M., Falomo, R., \& Treves, A. 1999b, ApJ, 526, 643

Scarpa, R., Urry, C. M., Padovani, P., Calzetti, D., \& O’Dowd, M. 2000b, ApJ, 544, 258

Schade, D. J., Boyle, B. J., \& Letawsky, M. 2000, MNRAS, 315, 498

Schlegel, D. J., Finkbeiner, D. P., \& Davis, M. 1998, ApJ, 500, 525

Sérsic, J.-L. 1968, Atlas de Galaxias Australes (Cordoba: Obs. Astron.)

Stickel, M., Fried, J. W., \& Kühr, H. 1993, A\&AS, 98, 393

Stocke, J. T., Morris, S. L., Gioia, J. M., et al. 1991, ApJS, 76, 813

Trujillo, I., Graham, A. W., \& Caon, N. 2001, MNRAS, 326, 869
Urry, C. M., Falomo, R., Scarpa, R., et al. 1999, ApJ, 512, 88

Urry, C. M., \& Padovani, P. 1995, PASP, 107, 803

Urry, C. M., Scarpa, R., O’Dowd, M., et al. 2000, ApJ, 532, 816

Villata, M., Raiteri, C. M., Lanteri, L., Sobrito, G., \& Cavallone, M. 1998, ApJS, 130, 305

Voges, W. 1992, in Proc. of the ISY Conference Space Science, ESA ISY-3 (ESA Publications), 9

Wright, S. C., McHardy, I. M., \& Abraham, R. G. 1998a, MNRAS, 295, 799

Wright, S. C., McHardy, I. M., Abraham, R. G., \& Crawford, C. S. 1998b, MNRAS, 296, 961

Wrobel, J. M., \& Lind, K. R. 1990, ApJ, 348, 135

Wu, X.-B., Liu, F. K., \& Zhang, T. Z. 2002, A\&A, 389, 742

Wurtz, R., Stocke, J. T., \& Yee, H. K. C. 1996, ApJS, 103, 109

Yanny, B., Jannuzi, B. T., \& Impey, C. 1997, ApJ, 484, L113 